\title{
[Fe II] jets from intermediate-mass protostars in Carina
}

\author{
Megan Reiter, ${ }^{\star \star}$ Nathan Smith ${ }^{2}$ and John Bally ${ }^{3}$ \\ ${ }^{1}$ University of Michigan, Ann Arbor, MI 48109, USA \\ ${ }^{2}$ Steward Observatory, University of Arizona, Tucson, AZ 85721, USA \\ ${ }^{3}$ Center for Astrophysics and Space Astronomy, University of Colorado, 389 UCB, Boulder, CO 80309, USA
}

Accepted 2016 September 14. Received 2016 September 7; in original form 2016 June 27

\begin{abstract}
We present new HST/WFC3-IR narrow-band [Fe II] images of protostellar jets in the Carina Nebula. Combined with five previously published sources, we have a sample of 18 jets and two Herbig-Haro (HH) objects. All of the jets we targeted with Wide-Field Camera 3 (WFC3) show bright infrared [ $\left.\mathrm{Fe}_{\mathrm{II}}\right]$ emission, and a few $\mathrm{H} \alpha$ candidate jets are confirmed as collimated outflows based on the morphology of their [Fe II] emission. Continuum-subtracted images clearly separate jet emission from the adjacent ionization front, providing a better tracer of the collimated jet than $\mathrm{H} \alpha$ and allowing us to connect these jets with their embedded driving sources. The [Fe II] $1.64 \mu \mathrm{m} / \mathrm{H} \alpha$ flux ratio measured in the jets is $\gtrsim 5$ times larger than in the adjacent ionization fronts. The low-ionization jet core requires high densities to shield $\mathrm{Fe}^{+}$ against further ionization by the FUV radiation from O-type stars in the $\mathrm{H}$ II region. High jet densities imply high mass-loss rates, consistent with the intermediate-mass driving sources we identify for 13 jets. The remaining jets emerge from opaque globules that obscure emission from the protostar. In many respects, the HH jets in Carina look like a scaled-up version of the jets driven by low-mass protostars. Altogether, these observations suggest that [Fe II] emission is a reliable tracer of dense, irradiated jets driven by intermediate-mass protostars. We argue that highly collimated outflows are common to more massive protostars, and that they suggest the outflow physics inferred for low-mass stars formation scales up to at least $\sim 8 \mathrm{M}_{\odot}$.
\end{abstract}

Key words: stars: formation.

\section{INTRODUCTION}

Understanding accretion and outflow in young stars is key to constraining the physics that govern their formation and early evolution. Accretion and outflow will shape the circumstellar environment around young stars where planet formation may already be ongoing, fostering or inhibiting the genesis of sub-stellar companions. Abundant low-mass sources in the solar neighbourhood allow for detailed, multiwavelength studies of the evolution of protostars from deeply embedded cores that are only accessible at long wavelengths (e.g. Enoch et al. 2008; Jørgensen et al. 2009) to the IR excess and strong optical emission lines characteristic of more evolved T Tauri stars (e.g. Evans et al. 2003; Harvey et al. 2007; Evans et al. 2009). For the nearby sources, high angular resolution observations can resolve the circumstellar geometry, clearly illustrating the importance of accretion discs, jets, and outflows for the formation of stars (e.g. Burrows et al. 1996; Krist et al. 1998; McCaughrean et al. 1998; Padgett et al. 1999).

In low-mass stars, disc material accretes along stellar magnetic field lines, ultimately splashing on to the stellar surface at high latitudes (see e.g. review by Bouvier et al. 2007). This process of

\footnotetext{
^E-mail: mreiter@umich.edu
}

magnetospheric accretion requires strong, large-scale stellar magnetic fields with a predominately dipolar topology to lift material from the inner edge of the disc and carry it along field lines to the star. Strong magnetic fields have been found in many T Tauri stars, supporting the magnetospheric accretion paradigm (e.g. Johns-Krull et al. 1999a, 2013; Johns-Krull, Valenti \& Koresko 1999b; JohnsKrull, Valenti \& Saar 2004). Whether a scaled-up version of this formation scenario applies to intermediate- and high-mass stars remains unclear. In particular, it is not settled whether intermediateand high-mass stars generate magnetic fields of sufficient strength to support magnetospheric accretion. Surveys of Herbig Ae/Be stars find a low magnetic incidence of $\leq 10$ per cent (see e.g. Wade et al. 2007). Derived upper limits on the magnetic field strength are smaller than the minimum field strength required for magnetospheric accretion in both Herbig Ae and Be stars (derived from the models of Johns-Krull et al. 1999b). More recent surveys with higher sensitivity to weaker fields further constrain the average field strength of intermediate-mass protostars to be an order of magnitude less than typically observed in T Tauri stars (e.g. Alecian et al. 2013; Hubrig et al. 2015).

Evidence for circumstellar discs around intermediate- and highmass protostars also argues for massive stars forming via a scaledup version of low-mass star formation (e.g. Tan \& McKee 2003). Direct detection is difficult, however, given the large median 
distances, high optical depths, and short time-scales involved (e.g. Beuther, Walsh \& Longmore 2009; Kraus et al. 2010; Preibisch et al. 2011; Carrasco-González et al. 2012; Johnston et al. 2015). For this reason, indirect accretion indicators provide an important avenue to understand the evolution of intermediate-mass protostars. Jets are one such signpost as they require active disc accretion. Observed similarities in the physical properties of outflows from low- and high-mass stars suggest a common production mechanism regardless of protostellar mass (Richer et al. 2000). Alternate formation pathways, for example the coalescence of lower-mass cores, are unlikely to form collimated outflows (Bally \& Zinnecker 2005). Thus, the detection of collimated jets provides compelling, though indirect, evidence of circumstellar discs (e.g. Guzmán et al. 2012).

Protostellar outflows appear to be a ubiquitous feature of star formation (e.g. Arce et al. 2007), and thus provide an avenue to identify accreting systems even at distances where the accretion geometry is not directly resolved. A well-collimated jet launched near the protostar requires energy from disc accretion to produce a collimated stream of high-velocity gas (e.g. Ferreira, Dougados $\&$ Cabrit 2006). An underlying jet, a wide-angle disc wind, or a combination of the two (e.g. Bachiller et al. 1995; Sandell et al. 1999; Arce \& Goodman 2001; Ybarra et al. 2006) may power the outflows typically studied at millimetre wavelengths. Outflows tend to have lower velocities and broader morphologies than the collimated jets that may be obscured inside the optically thick outflow lobe. This is especially true for high-mass protostars as they tend to form out of dense clumps that will birth a cluster of stars that have large columns of gas and dust that thoroughly enshroud the earliest evolutionary stages.

Comparatively little attention has been paid to intermediate mass ( 2-8 $\left.\mathrm{M}_{\odot}\right)$ stars, even though they sample the changes in stellar structure that may be related to a transition in accretion mechanisms between low- and high-mass stars. Unlike the highest mass sources, modest optical depths obscure intermediate-mass stars, permitting multiwavelength observations of a variety of spatial and temporal scales. Employing similar observational techniques as low-mass star formation makes it easier to directly compare the results (e.g. Calvet et al. 2004; Vink et al. 2005; Mendigutía et al. 2012). This potential to link low- and high-mass star formation has led to growing interest in intermediate-mass stars. However, studies typically target only a few jets or outflows from intermediate-mass stars, providing a heterogeneous sample of objects observed in different regions and with different techniques (e.g. Ellerbroek et al. 2013; Shepherd, Testi \& Stark 2003). Ideally, multiple sources in a single region would be observed with the same technique to develop a coherent picture of accretion and outflow throughout the intermediate-mass range.

H II regions offer one environment where jets from low- and intermediate-mass stars can be studied with similar techniques. Feedback from massive stars will have cleared much of the original molecular cloud, allowing UV radiation from nearby O-type stars to illuminate the jet body after it breaks free from its natal cloud. External irradiation lights up otherwise invisible components of the jet, including cold material that has not been excited in shocks and would therefore remain unseen in a quiescent region (see e.g. Reipurth et al. 1998; Bally \& Reipurth 2001). This more complete view allows for a better census of the mass in the jet. The physical properties of the jets can be calculated using the theory of photoionized gas, rather than complicated and time-dependent shock models (e.g. Bally et al. 2006). These jets are bright in many of the same lines (e.g. $\mathrm{H} \alpha,\left[\mathrm{S}_{\mathrm{II}}\right]$ ) as shock-excited Herbig-Haro $(\mathrm{HH})$ objects that are now known to be associated with protostellar outflows
(Herbig 1950, 1951; Haro 1952, 1953), and are therefore called $\mathrm{HH}$ jets. Many $\mathrm{HH}$ jets have been seen emanating from low-mass stars in Orion (Reipurth et al. 1998; Bally, O’Dell \& McCaughrean 2000; Bally \& Reipurth 2001; Bally et al. 2006), but few have been observed from intermediate-mass stars (e.g. Ellerbroek et al. 2013).

Smith, Bally \& Walborn (2010) discovered 39 jets and candidate jets in the Carina Nebula in an $\mathrm{H} \alpha$ imaging survey with HST/Advanced Camera for Surveys (ACS) that imaged many of the brighter regions in the nebula with indications of ongoing star formation. Jet mass-loss rates, estimated from the $\mathrm{H} \alpha$ emission measure (EM), are higher than those measured the same way for the jets in Orion, suggesting that the driving sources are intermediate-mass protostars. The high-luminosities of protostars identified along the jet axes support this hypothesis (see Smith, Bally \& Brooks 2004; Ohlendorf et al. 2012; Reiter \& Smith 2013).

Reiter \& Smith (2013) showed that bright [Fe II] emission from these jets arises in high density, low-ionization (or neutral) regions of the jets that are not bright in $\mathrm{H} \alpha$ emission. While [Fe II] is often assumed to be shock excited, this is not necessarily the case in regions with significant photoexcitation. Regardless of the excitation mechanism, however, the survival of $\mathrm{Fe}^{+}$emission in the harsh UV environment created by $\sim 70$ O-type stars in the Carina Nebula requires a large column of neutral material to shield $\mathrm{Fe}^{+}$ from further ionization by photons with energy $\geq 16.2 \mathrm{eV}$ (Reiter $\&$ Smith 2013). Less energetic photons will penetrate deeper into the jet, producing [Fe II] emission deeper in the jet core (the first ionization potential of $\mathrm{Fe}$ is $7.9 \mathrm{eV}$ ). Accounting for the neutral material in the jets increases the estimated mass-loss rate by as much as an order of magnitude, compared to that derived from the $\mathrm{H} \alpha$ EM. This points to a distinct class of powerful outflows from intermediate-mass protostars.

Several other factors point to near-IR [Fe II] emission as a better tracer of irradiated, high mass-loss rate jets in $\mathrm{H}_{\text {II }}$ regions. The irradiated pillars from which many of the jets emerge are themselves bright in $\mathrm{H} \alpha$, making it difficult to distinguish faint jet features from filamentary structures associated with the photoevaporative flow off the pillar. [Fe II] emission from the jet provides better contrast between the jet and environment (e.g. Smith et al. 2004), especially when offline continuum images exist to subtract photodissociation region (PDR) emission that might otherwise obscure faint jet features (see e.g. Reiter et al. 2015a). Near-IR [Fe II] emission helps penetrate the dusty birthplaces of the jet-driving protostars, connecting the larger-scale $\mathrm{H} \alpha$ outflow to the embedded IR source that drives it (see e.g. Smith et al. 2004; Reiter \& Smith 2013). In addition, $\mathrm{H} \alpha$ and [Fe II] emission trace different morphologies and kinematics near the protostar in some jets with embedded driving sources (Reiter et al. 2015a,b). When the two emission lines trace different outflow components, [Fe II] emission appears to be a better tracer of the protostellar jet, while the broader morphology and slower kinematics of $\mathrm{H} \alpha$ resemble those observed in entrained outflows.

In this paper, we present near-IR [Fe II] images obtained with HST/Wide-Field Camera 3 (WFC3)-IR of 18 jets, two HH objects, and one candidate jet in the Carina Nebula. We targeted 14 of the $\mathrm{HH}$ jets discovered by Smith et al. (2010) with a candidate driving source identified along the jet axis. Three additional candidate jets from Smith et al. (2010) and one new jet serendipitously fell within the imaged area. We confirm two of these as collimated jets based on their [Fe II] morphology. Combined with earlier [Fe II] imaging of four of the most powerful HH jets in Carina (Reiter \& Smith 2013), this provides a sample of 18 jets driven by sources throughout the $\sim 2-8 \mathrm{M}_{\odot}$ intermediate-mass range. 
Table 1. Observations.

\begin{tabular}{|c|c|c|c|c|c|}
\hline Target & $\alpha_{\mathrm{J} 2000}$ & $\delta_{\mathrm{J} 2000}$ & Date & Exp. time (s) & Comment \\
\hline HH 666 & 10:43:51.3 & $-59: 55: 21$ & 2009 Jul 24-29 & $2397 / 2797$ & No cont.; see also Reiter \& Smith (2013) \\
\hline HH 901 & 10:44:03.5 & $-59: 31: 02$ & 2010 Feb 1-2 & $2797 / 3197$ & No cont.; see also Reiter \& Smith (2013) \\
\hline HH 902 & 10:44:01.7 & $-59: 30: 32$ & 2010 Feb 1-2 & 2797/3197 & No cont.; see also Reiter \& Smith (2013) \\
\hline HH 1066 & $10: 44: 05.4$ & $-59: 29: 40$ & $2010 \mathrm{Feb} 1-2$ & $2797 / 3197$ & No cont.; see also Reiter \& Smith (2013) \\
\hline HH 900 & 10:45:19.3 & $-59: 44: 23$ & 2013 Dec 28 & 2397 & See also Reiter et al. (2015a). \\
\hline HH 903 & 10:45:56.6 & $-60: 06: 08$ & 2014 Apr 17 & 2397 & \\
\hline HH 1004 & $10: 46: 44.8$ & $-60: 10: 20$ & 2014 Sep 28 & 2397 & \\
\hline HH 1005 & $10: 46: 44.2$ & $-60: 10: 35$ & 2014 Sep 28 & 2397 & \\
\hline HH 1006 & $10: 46: 33.0$ & $-60: 03: 54$ & 2014 Jun 05 & 2397 & \\
\hline HH 1007 & $10: 44: 29.5$ & $-60: 23: 05$ & 2014 Apr 17 & 2397 & \\
\hline HН 1010 & $10: 41: 48.7$ & $-59: 43: 38$ & 2014 Apr 18 & 2397 & \\
\hline HH 1014 & $10: 45: 45.9$ & $-59: 41: 06$ & 2015 Jan 16 & 2397 & \\
\hline HH 1015 & 10:44:27.9 & $-60: 22: 57$ & 2014 Apr 17 & 2397 & \\
\hline $\mathrm{HH} \mathrm{c}-3$ & 10:45:04.6 & $-60: 03: 02$ & $2014 \mathrm{Feb} 20$ & 2397 & \\
\hline HH $1159^{*}$ & 10:45:08.3 & $-60: 02: 31$ & $2014 \mathrm{Feb} 20$ & 2397 & HH c-4 in Smith et al. (2010). \\
\hline HH $1160^{*}$ & 10:45:09.3 & $-60: 01: 59$ & 2014 Feb 20 & 2397 & HH c-5 in Smith et al. (2010). \\
\hline HH $1161^{*}$ & 10:45:09.3 & $-60: 02: 26$ & 2014 Feb 20 & 2397 & HH c-6 in Smith et al. (2010). \\
\hline HH $1162^{*}$ & 10:45:13.4 & $-60: 02: 55$ & 2014 Feb 20 & 2397 & HH c-7 in Smith et al. (2010). \\
\hline HH $1163^{*}$ & $10: 45: 12.2$ & $-60: 03: 09$ & 2014 Feb 20 & 2397 & HH c-8 in Smith et al. (2010). \\
\hline HH $1164^{*}$ & $10: 45: 10.5$ & $-60: 02: 42$ & 2014 Feb 20 & 2397 & New jet identified in this work. \\
\hline $\mathrm{HH} \mathrm{c}-10$ & 10:45:56.6 & $-60: 06: 08$ & 2014 Apr 17 & 2397 & \\
\hline HH $1156^{*}$ & $10: 45: 45.9$ & $-59: 41: 06$ & 2015 Jan 16 & 2397 & HH c-14 in Smith et al. (2010). \\
\hline
\end{tabular}

${ }^{*} \mathrm{HH}$ numbers are assigned by B. Reipurth in order to correspond with the catalogue of HH objects; see Reipurth, B. \& Reiter, M. 2017, A General Catalog of Herbig-Haro Objects, 3. Edition, in preparation.

\section{OBSERVATIONS}

\subsection{New [Fe II] images from $H S T /$ WFC3-IR}

Table 1 lists jet positions, observation dates and integration times of the new HST/WFC3-IR images presented in this paper obtained under programme GO-13391. Regions targeted with WFC3-IR contain one or more jets with a candidate driving source identified near the outflow axis. These candidate driving sources come from the Pan-Carina YSO Catalog (PCYC) identified and modelled by Povich et al. (2011). Using a total of eight pointings with HST, we obtained near-IR, narrow-band [Fe II] images and complementary offline continuum images for $16 \mathrm{HH}$ jets in Carina.

Observing these near-IR lines with $H S T$ allows us to obtain images free of the $\mathrm{OH}$ lines that contaminate narrow-band images obtained from the ground. Photon shot-noise dominates the signalto-noise ratio in ground-based data, severely limiting sensitivity to low surface brightness features. In addition, sky lines are variable, reducing the quality of images that isolate [Fe II] emission by subtracting an image of the offline continuum. The sensitivity and stability of $H S T$ are key to detailed study of jet emission, even though similar angular resolution is now possible with AO-fed 8-m telescopes on the ground.

For each pointing, we obtained images with a total integration time of $2397 \mathrm{~s}$ in the $F 126 N, F 130 N, F 164 N$, and $F 167 N$ filters. Observations employ the same setup and integration times used to obtain the [Fe II] images of the $\mathrm{HH} 666, \mathrm{HH}$ 901, $\mathrm{HH}$ 902, and HH 1066 jets in Carina presented by Reiter \& Smith (2013). We include those observations in this analysis. Relevant details of those images are listed in Table 1, and described in depth in Reiter \& Smith (2013). Together with new [Fe II] observations, this data set provides a sample with nearly uniform sensitivity to $[\mathrm{Fe}$ II] that allows us to detect faint jet features. Unlike the [Fe II] observations presented in Reiter \& Smith (2013), ${ }^{1}$ the new observations presented

\footnotetext{
${ }^{1}$ We analysed archival narrow-band [Fe II] images obtained with WFC3-IR after SM4 and to commemorate the 20th anniversary of HST.
}

in this paper also include simultaneous offline continuum images (in the $F 130 N$ and $F 167 N$ filters to complement $F 126 N$ and $F 164 N$, respectively). Using the same integration time in all four filters allows us to subtract continuum emission at the same signal to noise. All images were obtained using a box-dither pattern to avoid dead pixel artefacts and provide modest resolution enhancement.

\section{RESULTS}

We detect near-IR [Fe II] emission from every $\mathrm{HH}$ jet that we targeted in the Carina Nebula targeted for follow-up with HST/WFC3IR. Fig. 1 shows the distribution of the known O-type stars and $\mathrm{HH}$ jets in the Carina Nebula. Jets studied in this paper are denoted with thick black diamonds. We targeted a subset of jets with an embedded young stellar object (YSO) identified near the jet axis. Candidate jet-driving sources were selected from YSOs identified and modelled in the PCYC by Povich et al. (2011). In many cases, new [Fe II] observations confirm this source as the embedded protostar that drives the jet; these are marked with red squares in Fig. 1. Only for $\mathrm{HH} 900$ do [Fe II] observations definitively rule out the candidate driving sources identified near the outflow axis (see Section 3.2 and Reiter et al. 2015a). In the following sections, we briefly describe each jet.

\subsection{Jets without simultaneous offline continuum images (archival images)}

Reiter \& Smith (2013) presented narrow-band WFC3-IR [Fe II] images of four $\mathrm{HH}$ jets in Carina and demonstrated that bright [Fe II] emission traces low-ionization material in the shielded jet core. Two of the four jets studied by Reiter \& Smith (2013) have an IR-bright candidate protostar identified with Spitzer that lies near the jet axis. In both cases, [Fe II] emission traces the jet inside the natal globule, clearly connecting the larger scale $\mathrm{H} \alpha$ outflow with its embedded driving source. No emission from the jet is observed inside the globule from objects that do not have a point source detected near the jet axis with Spitzer. Instead, [Fe II] emission appears to be offset 


\section{RA [degrees]}

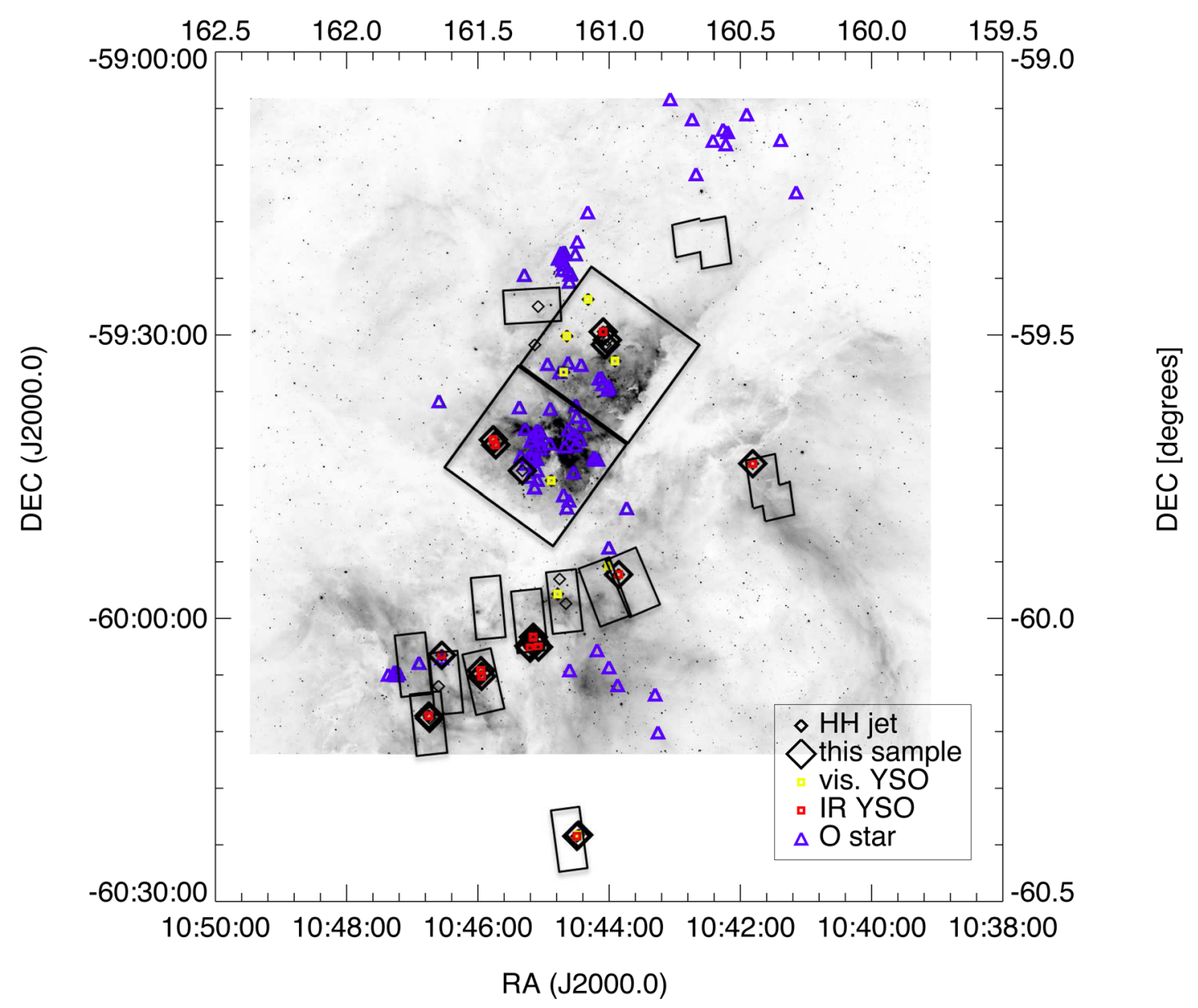

Figure 1. A ground-based $\mathrm{H} \alpha$ image of Carina with black diamonds that show the location of the HH jets discovered by Smith et al. (2010). Larger, thicker diamonds denote the subset of jets that we observed in [Fe II] with WFC3-IR. Red and yellow squares indicate jets with a driving source identified in the IR or optical, respectively. Blue triangles show the O-type stars in Carina catalogued by Smith (2006). Rectangles show the footprint of the original HST/ACS survey (also see fig. 1 in Smith et al. 2010).

$\sim 1 \operatorname{arcsec}(\sim 2000$ au or $\sim 0.01 \mathrm{pc}$ at the distance of Carina) from the globule edge. Below, we summarize key results for these four jets.

HH 666. The first jet detected in Carina was seen to have bright $\mathrm{H} \alpha$ and [Fe II] emission in ground-based images (Smith et al. 2004). Subsequent HST imaging showed that the two emission lines are offset laterally from one another. Bright [Fe II] emission traces a collimated jet that is surrounded by a cocoon of $\mathrm{H} \alpha$ emission. Taken together, the two lines show that HH 666 has the same morphology as a jet-driven outflow (see Fig. 2 and Smith et al. 2010; Reiter \& Smith 2013). Different kinematics traced by Doppler velocities in spectra of the two lines also argue for a two-component jet-outflow system. Fast, steady [Fe II] radial velocities more typical of a jet are surrounded by slower $\mathrm{H} \alpha$ emission that accelerates away from the driving source, similar to the Hubble flows seen in entrained molecular outflows (Smith et al. 2004; Reiter et al. 2015b).

HH 901 and HH 902. Smith et al. (2010) hypothesized that HH 901 and HH 902 are both high-density jets with an unseen neutral core based on sharp $\mathrm{H} \alpha$ emission that traces the ionization front along the length of the jets. Near-IR [Fe II] observations presented in Reiter \& Smith (2013) demonstrate that the ionized gas traced by $\mathrm{H} \alpha$ does not sample the full width of the jet. Bright [Fe II] emission peaks behind the $\mathrm{H} \alpha$, further away from the ionizing source, consistent with an ionized skin $(\mathrm{H} \alpha)$ that shields the neutral jet core ([Fe II], see Figs 3 and 4). Neither HH 901 nor HH 902 has a driving source detected in the globule, although proper motions require a source located near the head of the pillar in both cases (Reiter \& Smith 2014).

HH 1066. Smith et al. (2010) identified HH 1066 as a candidate jet based on streamers of $\mathrm{H} \alpha$ emission coming off the globule that point towards a bow shock. No unambiguous jet body could be identified in $\mathrm{H} \alpha$ images alone. However, bright [Fe $\mathrm{II}]$ emission clearly traces a collimated jet body that bisects the Spitzer-identified YSO (see Fig. 5 and Reiter \& Smith 2013). The ratio of the two [Fe II] lines shows that the reddening increases towards the inner jet, reaching a maximum at the point source, likely tracing the edge-on optically thick circumstellar disc.

\section{2 [Fe II] observations with simultaneous offline continuum subtracted}

HH 900 (previously published). As in HH 666, HH 900 shows a bright, bipolar [Fe II] jet with fast, steady velocities moving away from the globule. The narrow jet traced by near-IR [Fe II] emission plows through a broader, slower $\mathrm{H} \alpha$ outflow (see Fig. 6 and 

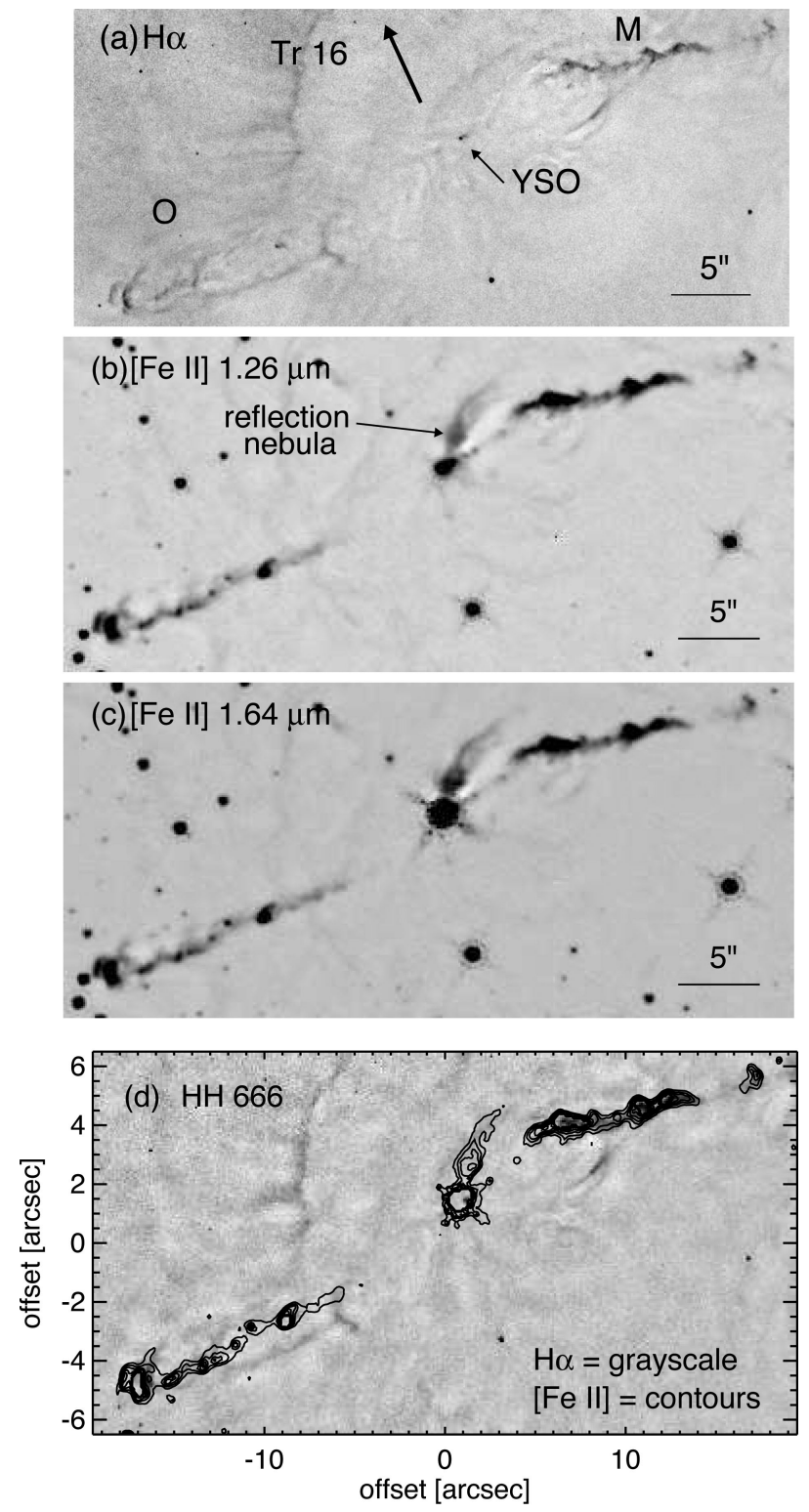

Figure 2. $\mathrm{H} \alpha$ (a) and [ $\mathrm{Fe} \mathrm{II}](\mathrm{b}, \mathrm{c})$ images of $\mathrm{HH} 666$ from Reiter \& Smith (2013). These archival [Fe II] data were not obtained with simultaneous offline continuum images. Each image has been median-filtered to improve contrast with emission from the irradiated dust pillar. Panel (d) shows [Fe II] contours $(1.26 \mu \mathrm{m}+1.64 \mu \mathrm{m})$ on the HST/ACS H $\alpha$ image. HH 666 is an example of a two-component jet, with wide-angle $\mathrm{H} \alpha$ emission that surrounds the collimated [Fe II] jet. Straight near-IR [Fe II] emission inside the pillar clearly connects the larger scale jet with the driving protostar.

Reiter et al. 2015a). $\mathrm{H} \alpha$ (and $\mathrm{H}_{2}$ ) emission from the outflow extend continuously from the edge of the globule. In contrast, continuumsubtracted $[\mathrm{Fe}$ II] images clearly show that $[\mathrm{Fe}$ II] emission from the jet is offset from the edge of the globule by $\sim 1$ arcsec (and not simply confused with bright emission from the PDR, along the surface of the globule). The jet axis defined by the [Fe II] emission bisects the globule and $\mathrm{H} \alpha$ proper motions require a driving source embedded in the dark, tadpole-shaped globule. However, no point source has been detected inside the globule in available IR images.

$H H$ 903. Fig. 7 shows HH 903 bursting from the western edge of a dust pillar in the actively star-forming south pillars (Smith et al. 2010). $\mathrm{H} \alpha$ emission from the jet extends on either side of the
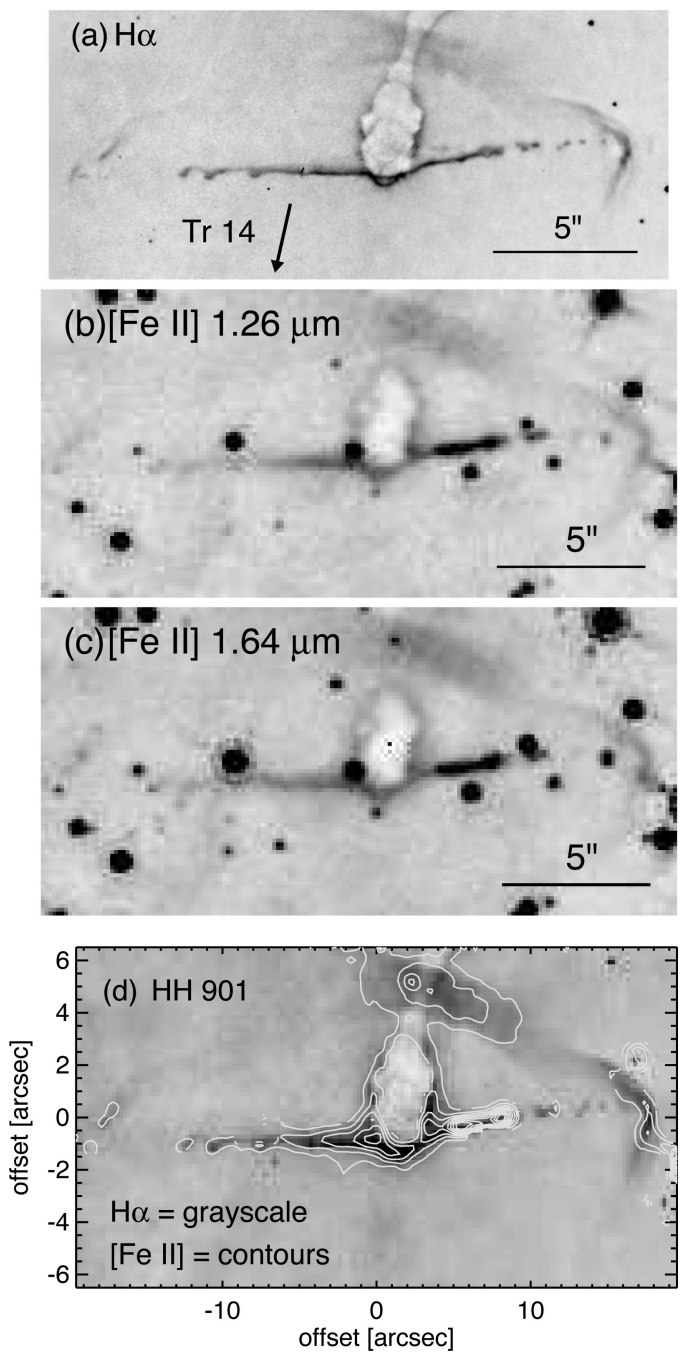

Figure 3. $\mathrm{H} \alpha$ (a) and [Fe II] (b,c) images of HH 901 from Reiter \& Smith (2013). Panel (d) shows contours [Fe II] emission on an $\mathrm{H} \alpha$ image. The bright star seen in the near-IR images at the eastern edge of the pillar is an interloper, as it lies too far above the [Fe II] jet axis to be the driving source. Kinematics require a driving source embedded in the head of the globule, although no protostar has been detected.

pillar, reaching a total projected length of $\sim 2 \mathrm{pc}$. The collimated jet identified in $\mathrm{H} \alpha$ images propagates west, delineating a jet axis perpendicular to the major axis of the pillar. To the east, $\mathrm{H} \alpha$ emission is much less collimated, with tenuous streams of emission that trace a broader sheath like those seen in HH 900 and HH 666 O.

In new WFC3-IR images, [Fe II] emission traces the collimated western jet limb seen in $\mathrm{H} \alpha$ and delineates the narrow stream of the eastern jet. Unlike $\mathrm{H} \alpha$, [Fe II] from the inner jet reaches inside the dust pillar, connecting the larger jet to an embedded IR source. The putative driving source lies immediately inside the ionization front along the western edge of pillar. The point source can clearly be seen in Spitzer/IRAC images with $\lambda \geq 4.5 \mu \mathrm{m}$, but is not detected at enough wavelengths to be included among the high-probability YSOs cataloged in the PCYC by Povich et al. (2011). However, Ohlendorf et al. (2012) model the mid-IR emission and estimate a protostellar mass of $\sim 4 \mathrm{M}_{\odot}$ (see Table 3 ). As the jet breaks into the $\mathrm{H}$ II region, it disrupts the bright ionization front along the pillar edge, similar to HH 1016 (see Smith et al. 2010). 


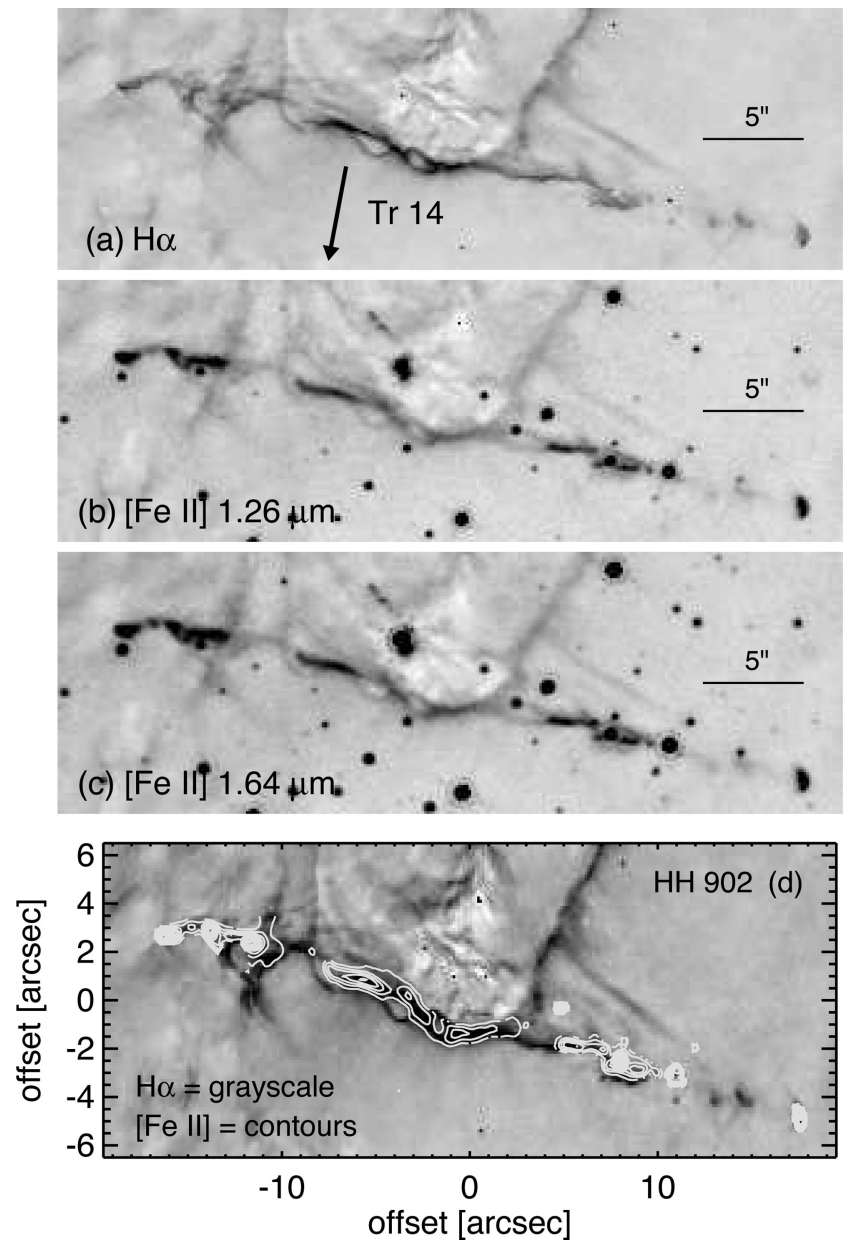

Figure 4. $\mathrm{H} \alpha$ (a) and [Fe II] (b,c) images of $\mathrm{HH} 902$ from Reiter \& Smith (2013), with a comparison of the two in panel (d). Bright [Fe II] emission traces the body of the jet along the edge of the broad natal pillar. Like $\mathrm{HH}$ 901, no driving source has been detected but kinematics require a protostar just inside the apex of the pillar.

To the east, $[\mathrm{Fe}$ II] emission traces a single jet axis that bends into an ' $S$ ' shape as it propagates away from the driving source. Bright knots of [Fe II] bisect more diffuse $\mathrm{H} \alpha$ emission, similar to HH 666 O (see Reiter et al. 2015b). This [Fe II]-jet and H $\alpha$-cocoon morphology has been seen in other HH jets in Carina (e.g. HH 900 and HH 666, see Reiter et al. 2015a,b). Further east, two bright [Fe II] knots may be part of the larger bow-shock structure seen in $\mathrm{H} \alpha$.

HH 1004. HH 1004 emerges from the head of a broad dust pillar. Like HH 903 and $\mathrm{HH}$ 1016, $\mathrm{H} \alpha$ emission along the pillar edge is clumpy, illustrating the disruption of the ionization front by the passage of the jet. To the south-west, a diffuse bow shock structure seen in $\mathrm{H} \alpha$ lies in front of the pillar, tracing the blueshifted limb of the jet.

Bright $\left[\mathrm{Fe}_{\mathrm{II}}\right]$ traces the collimated jet to the north-east and points to the apex of the bow shock to the south-west (see Fig. 8). A continuous stream of [Fe II] emission from the north-east connects the jet to the Spitzer-detected driving source (PCYC 1198, see Table 3 and Povich et al. 2011). Inside the pillar, to the south-west of the driving source, an arc of [Fe II] emission may trace the shock created as the counterjet emerges from the pillar. Outside this point, [ $\mathrm{Fe} \mathrm{II}]$ traces a narrow jet body that hooks upward, terminating in the centre of the bow shock. [Fe II] emission is brightest at the head of the bow shock while $\mathrm{H} \alpha$ emission traces the larger structure including the
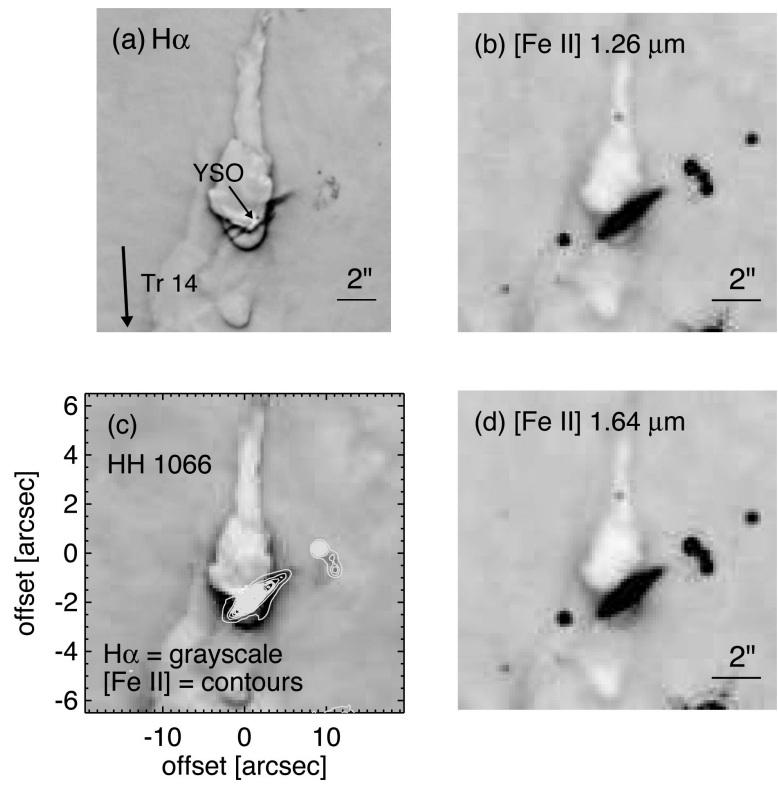

Figure 5. $\mathrm{H} \alpha$ (a) and $[\mathrm{Fe} \mathrm{II}](\mathrm{b}, \mathrm{c})$ images of $\mathrm{HH} 1066$ from Reiter \& Smith (2013). Strong, collimated [Fe II] emission confirmed the jet-like nature of HH 1066 (see panel d). Two spots of $\mathrm{H} \alpha$ emission trace where the jet breaks out of the pillar (see panel a), tracing a bipolar outflow from a young, Spitzer-identified protostar located at the tip of the globule.

extended bow shock wings. As in HH 666 and HH 903, H $\alpha$ appears to trace a cocoon enveloping the $[\mathrm{Fe} I \mathrm{I}]$ jet.

HH 1005. Further south in the same pillar as HH 1004 lies HH 1005. Smith et al. (2010) identified a bright, collimated $\mathrm{H} \alpha$ jet that points towards the south-east. No obvious bow shocks indicate more distant portions of the outflow. Smith et al. (2010) propose that, like $\mathrm{HH} 901$ and $\mathrm{HH}$ 902, bright $\mathrm{H} \alpha$ emission in $\mathrm{HH} 1005$ traces an ionization front in a high-density jet. Indeed, new WFC3-IR images reveal $[\mathrm{Fe} \mathrm{II}]$ emission behind the $\mathrm{H} \alpha$ stream, tracing the neutral jet core that must be shielded by a large column of material. However, $\mathrm{H} \alpha$ and $[\mathrm{Fe} \mathrm{II}]$ emission are clearly offset $(\sim 1 \mathrm{arcsec})$ from each other, similar to the distinct $\mathrm{H} \alpha$ and $[\mathrm{Fe}$ II] features seen in $\mathrm{HH} 900$ and HH 666 (see Reiter et al. 2015a,b). The position angle of the jet axis inferred from [ $\mathrm{Fe}$ II] emission differs from that measured in $\mathrm{H} \alpha$ images by Smith et al. (2010) by $\sim 17^{\circ}$. The $\mathrm{H} \alpha$ emission resembles an arm extending off the pillar edge while [Fe $\mathrm{II}]$ emission is more closely aligned with a chain of [Fe II] knots that bisect the width of the pillar and extend past the western edge.

Several discrete [Fe $\mathrm{FI}$ knots not seen in $\mathrm{H} \alpha$ trace the jet axis through the pillar. Most knots fall along a single, smooth stream. This is not the case where the jet emerges from the western edge of the pillar. Two streams separated by $\sim 5$ arcsec lie above and below the jet axis defined by the rest of the $[\mathrm{Fe} I I]$ knots. Where the jet emerges from the pillar, the opening angle between the two streams is $\sim 20^{\circ}$, however this narrows as the jet moves further into the $\mathrm{H}$ II region. $\mathrm{H} \alpha$ emission from $\mathrm{HH} 900$ shows a similar narrowing of the outflow morphology with increasing distance from the source (see Fig. 6 and Reiter et al. 2015a). To the west, outside the pillar, [Fe II] knots coincide with two smooth wisps of $\mathrm{H} \alpha$ separated by $\sim 3.5$ arcsec that Smith et al. (2010) identified as part of the HH 1004. However, these features lie along the $\mathrm{HH} 1005$ jet axis defined by [Fe II]. Unlike other jets with broad $\mathrm{H} \alpha$, the [Fe II] emission does not resemble a collimated jet body surrounded by an $\mathrm{H} \alpha$ cocoon. Instead, [Fe II] knots trace an arc between the two wisps of $\mathrm{H} \alpha$ emission. 

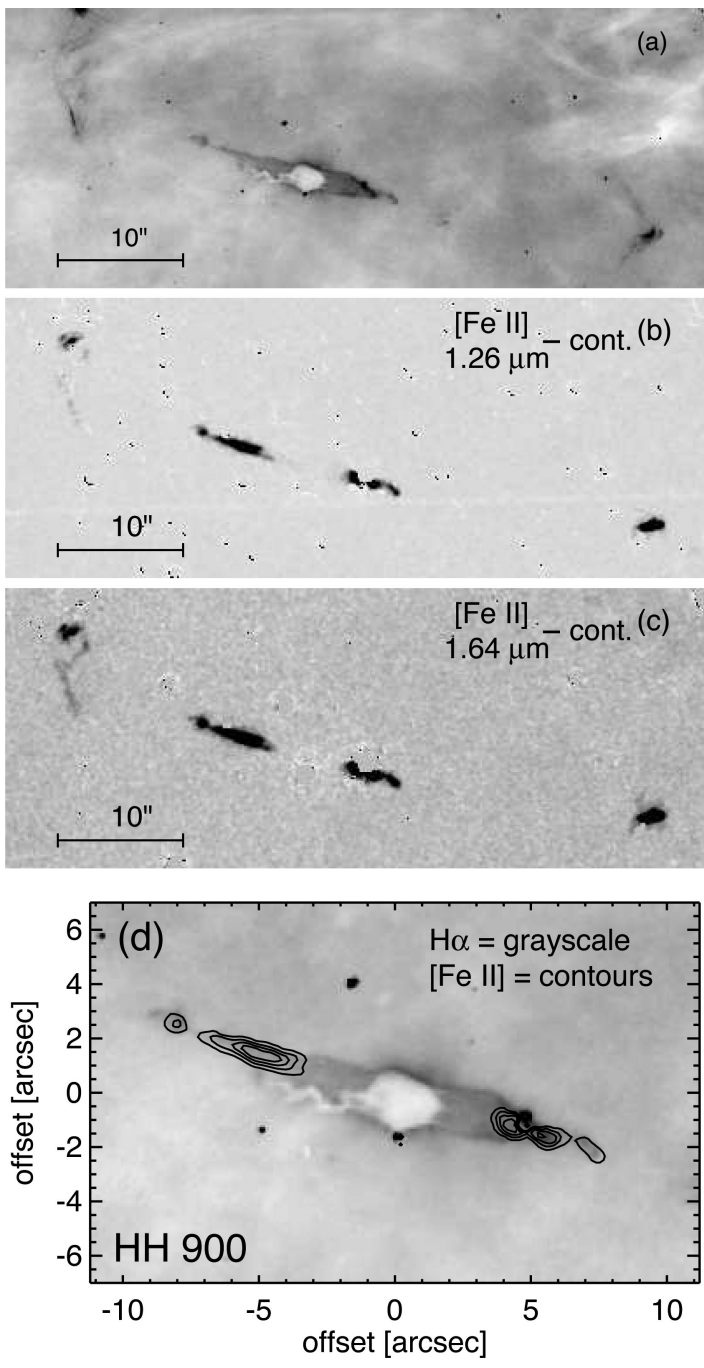

Figure 6. H $\alpha$ image (a) of HH 900 from Smith et al. (2010) and continuumsubtracted [Fe II] images (b,c) previously published in Reiter et al. (2015a). $\mathrm{HH} 900$ is one of the two-component jets seen in Carina where $\mathrm{H} \alpha$ emission traces the wide-angle, lower velocity outflow and $\left[\mathrm{Fe}_{\mathrm{II}}\right]$ traces the faster, collimated jet (d).

Curiously, neither HH 1004 or HH 1005 point towards the $\mathrm{H} \alpha$ bow shock that Smith et al. (2010) identified east of the pillar. A separate arc of [Fe II] emission lies to the south-west of both jets, but not clearly along the axis of either. Proper motions are required to determine if either feature is physically associated with these jets.

HH 1006. Like HH 900, HH 1006 was first identified in groundbased images as a candidate proplyd by Smith, Bally \& Morse (2003). H $\alpha$ images from HST clearly reveal a collimated bipolar jet emerging from a small cometary cloud (Smith et al. 2010). The jet axis of HH 1006 is aligned parallel to the major axis of the cloud, providing a counterexample to the many jets that are seen perpendicular to the long axis of their natal pillars (see e.g. Raga, Lora \& Smith 2010). Sahai, Güsten \& Morris (2012) estimate a globule mass of $0.35 \mathrm{M}_{\odot}$ from molecular line observations (with 8-19 arcsec resolution, depending on the tracer) and identify the IR-bright driving source embedded in the globule.

Fig. 9 shows that $[\mathrm{Fe} \mathrm{II}]$ and $\mathrm{H} \alpha$ trace the same jet morphology outside the cloud. [Fe II] emission from the northern limb of the jet extends inside the globule and connects the jet to IR-bright driving source (PCYC 1172, see Table 3 and Povich et al. 2011). This suggests that the northern limb of the jet is tilted slightly towards the observer (blueshifted), providing a clear view of the jet all the way to the driving protostar. In contrast, [Fe II] emission from the southern lobe of the jet begins where there appears to be a notch taken out of the south-east side of the globule, $\sim 3$ arcsec south of the putative driving source.

New [Fe II] images also reveal a bow shock to the north of $\mathrm{HH}$ 1006 (the northern bow shock lies beyond the edge of the $\mathrm{H} \alpha$ image; see Fig. 9), complementing the bow shock found to the south of the jet by Smith et al. (2010). The northern and southern bow shocks are both offset from the driving source by $\sim 30$ arcsec, making the total outflow length $\sim 1 \mathrm{pc}$, twice as long as the $\sim 0.45 \mathrm{pc}$ length estimated by Smith et al. (2010).

HH 1007 and HH 1015. Smith et al. (2010) identify HH 1007 as a dense jet body in the saddle between two neighbouring dust pillars. HH 1007 emerges from the western edge of the eastern pillar and points towards the neighbouring cloud. Only the brightest $\mathrm{H} \alpha$ knot located between the dark dust clouds shows [Fe $\mathrm{II}$ ] emission (see Fig. 10). [Fe II] emission breaks up into a bright, central knot with a smaller knot extending to the north-west. These knots lie immediately next to an unobscured star located between the pillars. However, this star is not in the PCYC and likely not related to $\mathrm{HH}$ 1007. There is no evidence for collimated [Fe II] emission from any other part of $\mathrm{HH} 1007$.

HH 1015 resides in the same cloud complex, emerging from the tip of the western of the two pillars. Like HH 1006, HH 1015 does not emerge perpendicular to the major axis of the pillar. Instead, the jet axis makes a $47^{\circ}$ angle with the major axis of the pillar. Faint $\mathrm{H} \alpha$ emission reveals a collimated jet body that extends $\sim 6$ arcsec into the $\mathrm{H}_{\text {II }}$ region.

Like $\mathrm{H} \alpha,[\mathrm{Fe} I I]$ is faint, but clearly traces the collimated jet. An IR-bright YSO lies just inside the pillar edge at the base of HH 1015, and is the likely driving source (PCYC 538; Povich et al. 2011). Extrapolating the jet axis defined by $\mathrm{H} \alpha$ and [Fe II] to estimate the position of the counterjet, we find that the south-east limb HH 1015 may intersect $\mathrm{HH}$ 1007. In fact, the knots in $\mathrm{HH} 1007$ all lie along the $\mathrm{HH} 1015$ jet axis, but are offset from the $\mathrm{H} \alpha$ emission that Smith et al. (2010) identified as HH 1007.

HH 1010. $\mathrm{H} \alpha$ emission traces a bipolar jet emerging perpendicular to the major axis of a pillar head (Smith et al. 2010). The two limbs appear symmetric, tracing a jet that extends $\sim 15$ arcsec beyond the pillar edge. Smith et al. (2010) identify a putative bow shock, HH $1010 \mathrm{~A}$, to the south-west, suggesting a total jet length $\gtrsim 60$ arcsec.

Our new WFC3-IR images show that bright [Fe II] emission in $\mathrm{HH} 1010$ delineates the same collimated jet morphology seen in $\mathrm{H} \alpha$ images (see Fig. 11). As with $\mathrm{HH}$ 1006, [Fe II] emission extends inside the dust pillar, connecting the extended $\mathrm{H} \alpha$ outflow with the embedded IR driving source (PCYC 55, see Table 3 and Povich et al. 2011). The driving source does not lie halfway between the two $\mathrm{H} \alpha$ jets limbs, but closer to the north, roughly $1 / 3$ of the way into the pillar. The YSO and the beginning of the northern jet limb coincide with the position of the faint optical source noted within the boundaries of the pillar by Smith et al. (2010). [Fe II] emission from the southern limb of HH 1010 is offset from the driving source, beginning $\sim 6$ arcsec away. This geometry suggests that the southern limb of HH 1010 is redshifted and the northern limb blueshifted.

A few additional $[\mathrm{Fe}$ II] knots lie to the south of the pillar. Several knots trace a backward ' $\mathrm{L}$ ' shape and can also be seen in $\mathrm{H} \alpha$ images. These knots lie $\sim 9$ arcsec to the north-west of HH 1010 A, but closer to the jet axis defined by the [Fe II]. The stem of the backward ' $\mathrm{L}$ ' lies along the jet axis, while the foot may trace the 

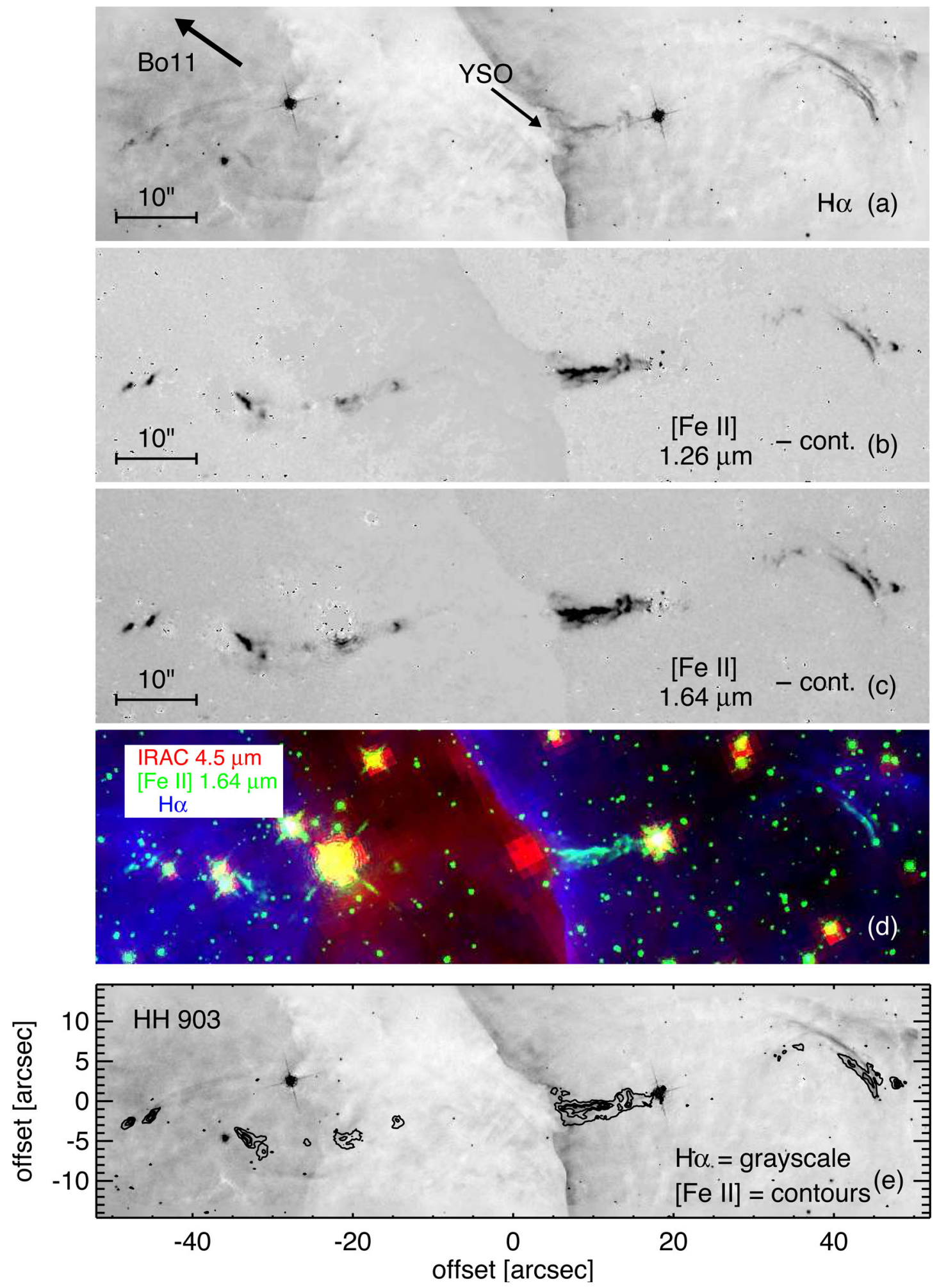

Figure 7. The $\mathrm{H} \alpha$ image (a) from Smith et al. (2010) is shown alongside new, continuum-subtracted near-IR [Fe II] images (b,c) of HH 903. [Fe II] emission connects the large-scale jet to the IR-bright protostar that lies just inside the pillar ionization front as shown in panel (d). Contours of [Fe II] emission on an $\mathrm{H} \alpha$ image (e) highlight portions of the jet inside the pillar that are only seen in the IR.

culminating bow shock. We do not detect [Fe II] emission from $\mathrm{HH}$ $1010 \mathrm{~A}$.

HH 1014. HH 1014 emerges from the head of a dust pillar in the dark molecular ridge that borders the bright inner part of the Carina
Nebula. Like HH 1006, the axis of the jet is aligned to within a few degrees of the pillar semimajor axis. No counterjet can be seen in $\mathrm{H} \alpha$ images. Both HH 1014 and HH 900 lie close to $\eta$ Carinae. However the two jets have very different morphologies. While $\mathrm{H} \alpha$ emission 

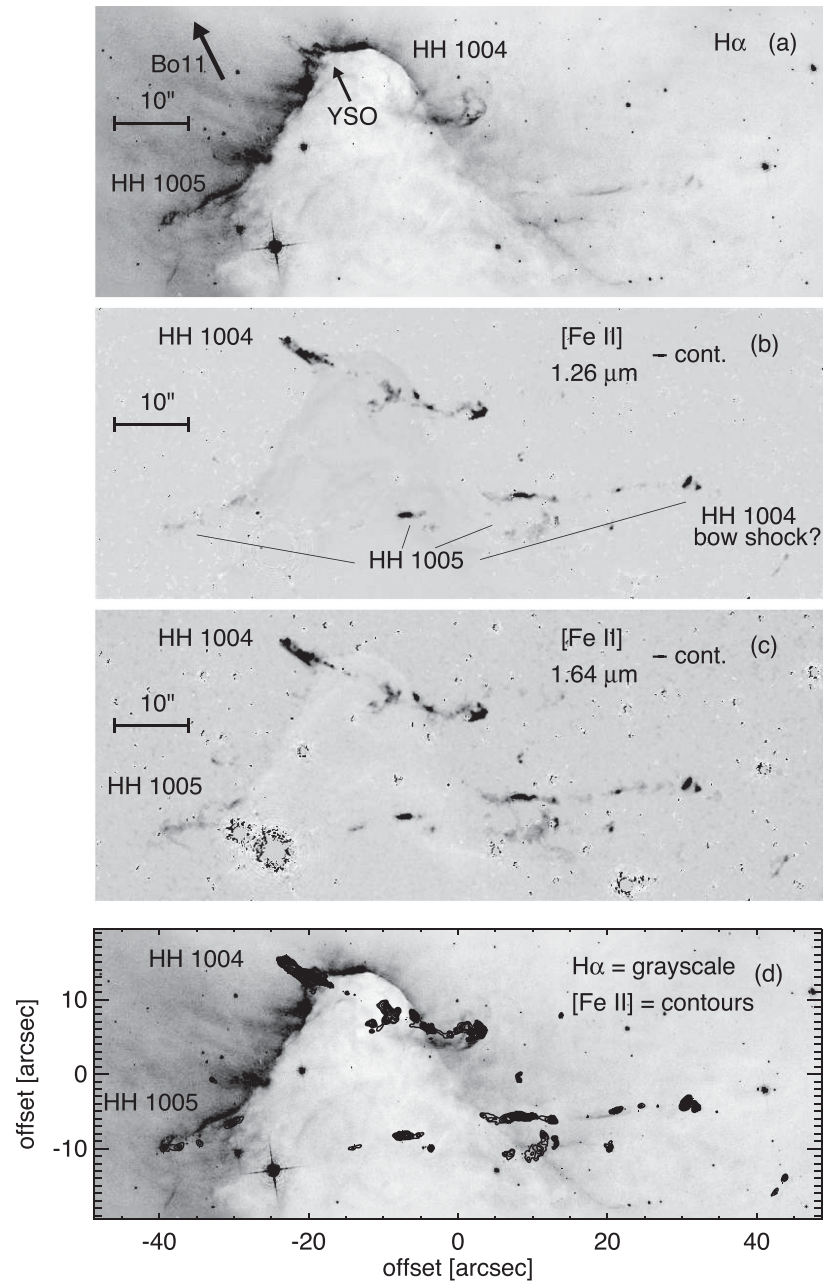

Figure 8. H $\alpha$ image (a) from Smith et al. (2010) shows two jets, HH 1004 and $\mathrm{HH} 1005$, emerging from a dust pillar. New, continuum-subtracted [Fe II] images $(b, c)$ show the collimated, bipolar body of HH 1004. Several [Fe II] knots trace out $\mathrm{HH} 1005$ as it extends through the pillar (d) and into the $\mathrm{H}_{\text {II }}$ region on either side. Unlike $\mathrm{HH} 1004$, the morphology of the [Fe II] emission in $\mathrm{HH} 1005$ does not clearly identify the driving protostar.

from $\mathrm{HH} 900$ traces the wide-angle stream of an entrained outflow, $\mathrm{H} \alpha$ emission from $\mathrm{HH} 1014$ clearly traces a narrow, collimated jet.

New [Fe II] images shown in Fig. 12 show bright emission from the collimated jet and confirm that it is driven by a protostar at the tip of the pillar (PCYC 984; Povich et al. 2011). Inside the pillar, another $[\mathrm{Fe}$ II] knot lies along the same axis, $\sim 5 \operatorname{arcsec}$ to the east of the driving source, likely tracing the counterjet. Two additional [Fe II] knots located further east may also be part of HH 1014. However, these two knots are offset slightly north of the jet axis. Other jets in Carina have been observed to bend towards (e.g. HH 900) or away (HH 901) from nearby O-type stars (see also Smith et al. 2010; Reiter \& Smith 2013; Reiter et al. 2015a).

$H H$ 1156. Smith et al. (2010) noted an H $\alpha$ feature with shocklike morphology that lies to the south-west of the pillar housing $\mathrm{HH}$ 1014. The shock does not lie along the HH 1014 jet axis and is instead identified as candidate jet HH c-14. Smith et al. (2010) suggest that it may be driven by an IR source embedded in another pillar in the region.

New WFC3-IR images reveal [Fe II] emission from $\mathrm{HH}$ c-14. More striking is the bright well-collimated [Fe II] jet that emerges from the dust pillar 27 arcsec to the north-east of the HH c-14 bow
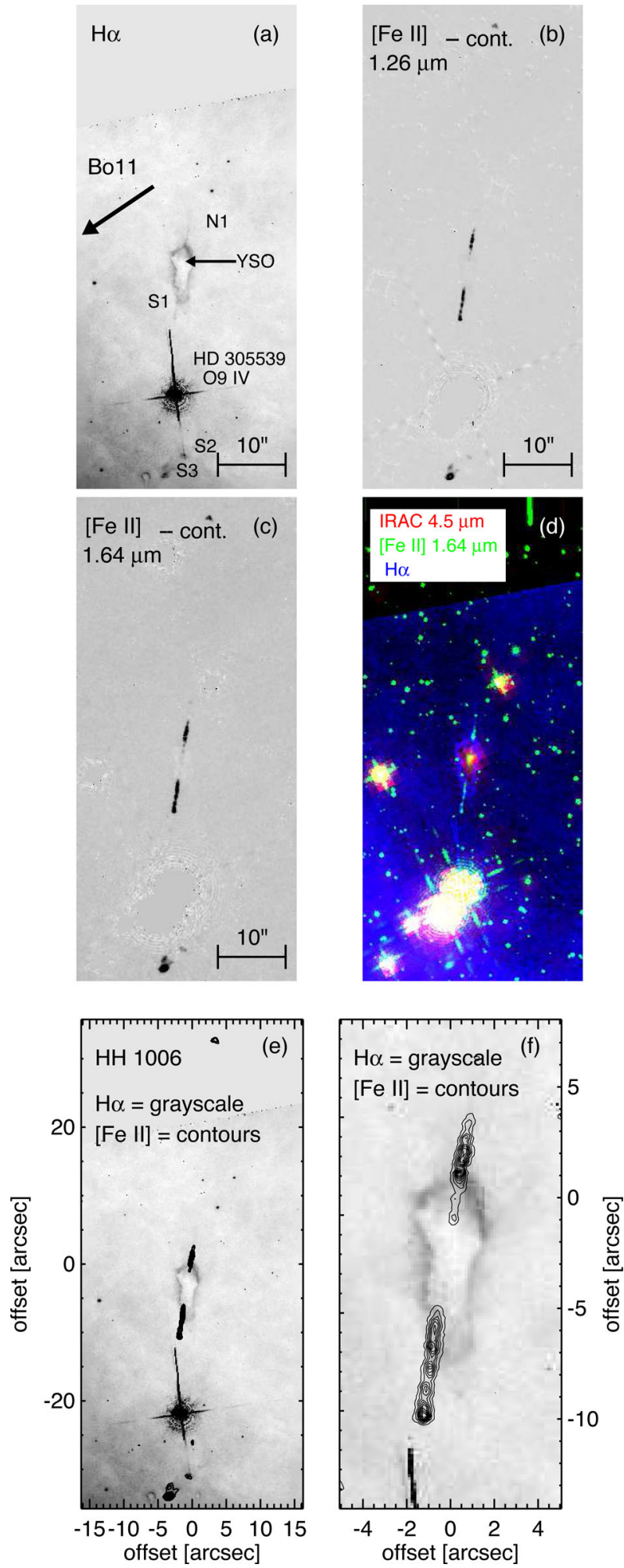

Figure 9. Like $\mathrm{H} \alpha$ (from Smith et al. 2010, shown in panel a), [Fe II] emission (b,c,d,e) from HH 1006 traces a highly collimated bipolar jet. $\mathrm{HH}$ 1006 is one of the few examples of a jet that is aligned nearly parallel to the major axis of its natal globule. Panel (f) shows a zoom of the highly collimated $[\mathrm{Fe} \mathrm{II}]$ jet emerging from the small globule. 

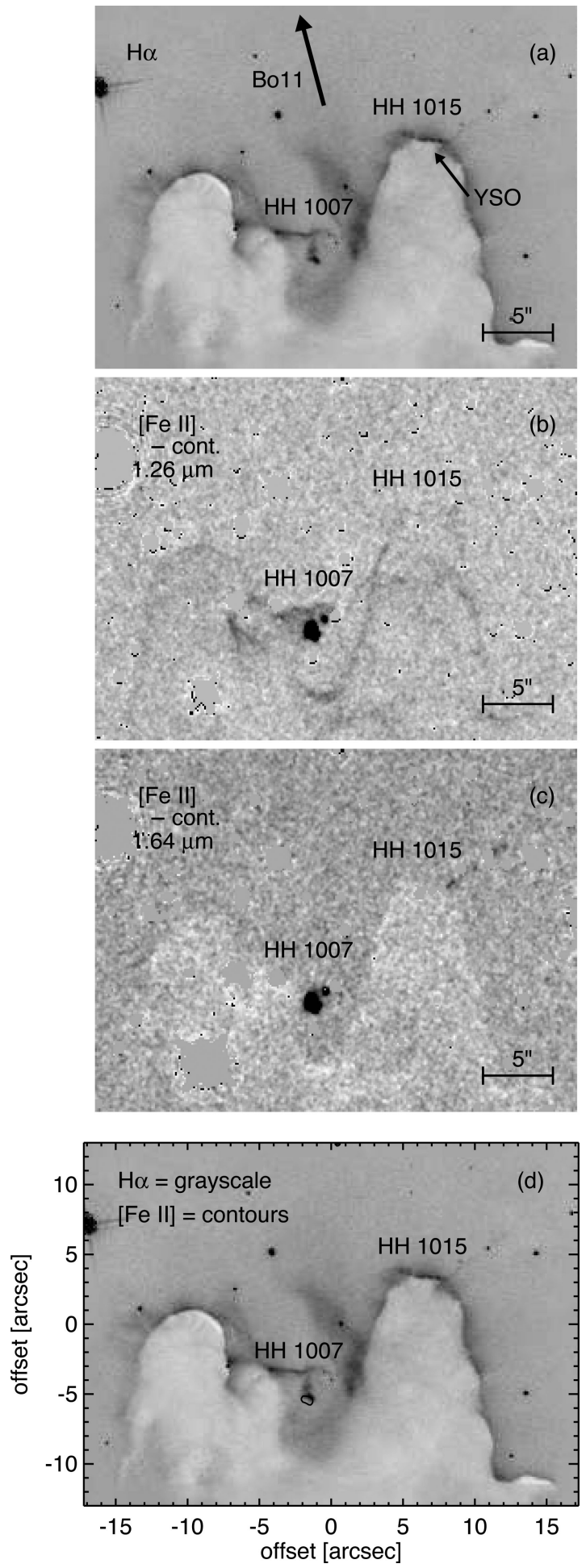

Figure 10. $\mathrm{H} \alpha$ (a) and new [Fe II] (b,c) images of the twin pillars that house HH 1007 (near the centre of the image) and HH 1015 (top right). HH 1015 shows the weakest [Fe II] of all the jets in this sample, unlike the bright knots of [Fe II] emission seen in $\mathrm{HH} 1007$ (d).

shock. This bipolar jet lies at the tip of a pillar immediately south of HH 1014 and points towards the putative bow shock. H $\alpha$ images offer no hint of a jet at the head of this pillar. Nevertheless, the jet appears to emerge from an unobscured and IR-bright protostar (PCYC 986; Povich et al. 2011). Additional [Fe II] knots lie along
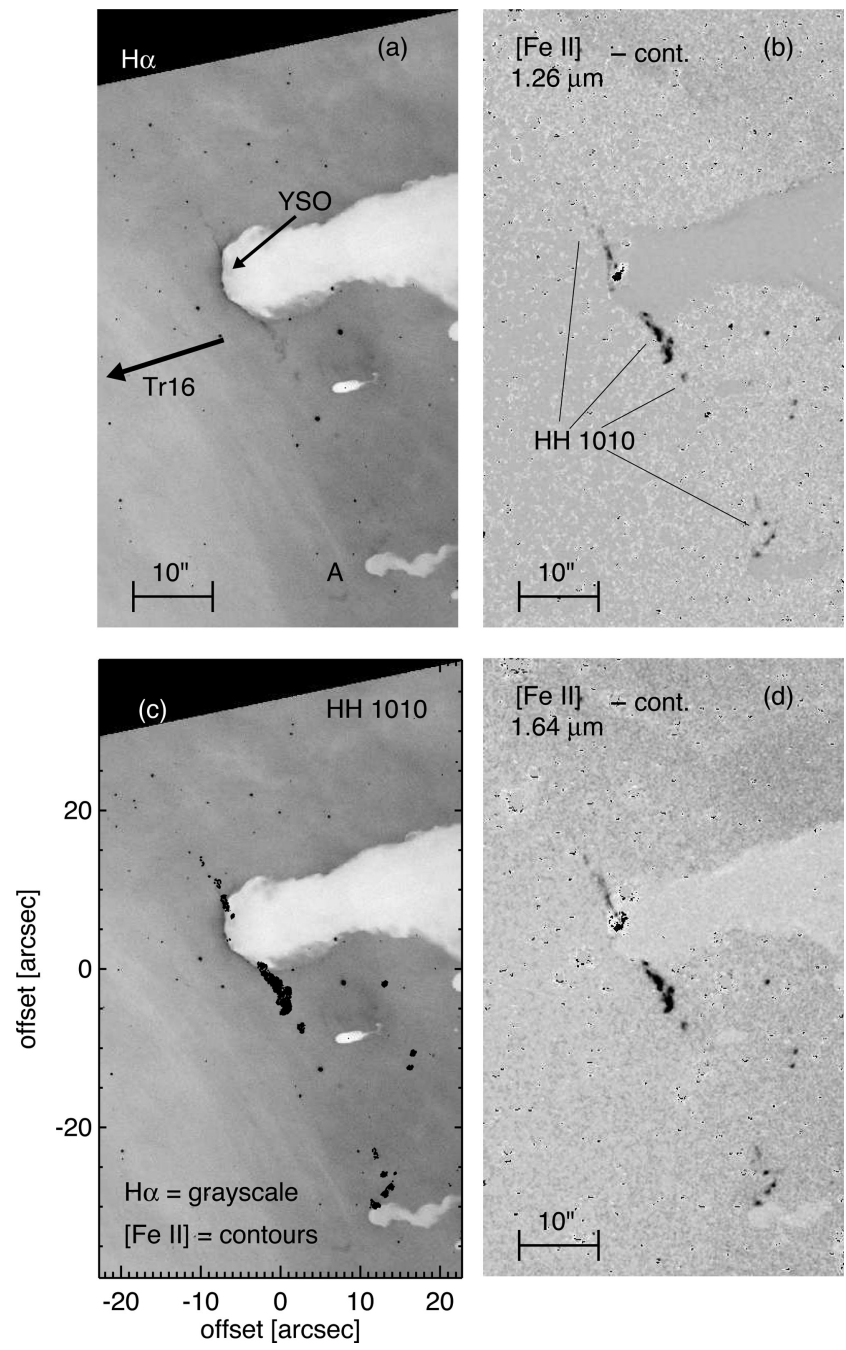

Figure 11. HH 1010 is a bipolar jet that emerges from the head of a dust pillar, as seen in $\mathrm{H} \alpha$ (a). [Fe II] (b,d) emission from the jet extends inside the pillar (c), connecting the larger-scale jet to the Spitzer-identified driving source.

the jet axis to the north-east and may be the complementary bow shock. Both putative bow shocks lie slightly offset from the axis defined by the inner jet, but altogether may trace an S-shaped jet. Given the well-defined jet morphology seen in new [Fe II] images, we assign this jet an $\mathrm{HH}$ number of 1156 .

HH $c$-3. Multiple protrusions extend off the head of a cometary cloud tracing candidate jet (or jets) HH c-3. No bow shocks are found nearby to indicate the outflowing nature of the gas. Extremely faint $\left[\mathrm{Fe}_{\mathrm{II}}\right]$ emission from $\mathrm{HH}$ c-3 B traces the same morphology seen in $\mathrm{H} \alpha$ (see Fig. 13). An IR-bright point source is detected near the apex of the cloud, consistent with the position of the base of the putative jet. With no velocity information and the poor signal to noise achieved near the edge of the frame where $\mathrm{HH} \mathrm{c-3}$ is located, it remains unclear whether $\mathrm{HH} \mathrm{c}-3$ is, in fact, a jet.

HH 1159 through 1164. Smith et al. (2010) identified several $\mathrm{H} \alpha$ knots clustered around an extended globule in the south pillars. Five separate $\mathrm{HH}$ objects and jet-like features likely trace multiple outflows, although the relationship between each feature is unclear from $\mathrm{H} \alpha$ images alone. New [Fe II] images shown in Fig. 14 reveal a network of collimated jets that reach inside the pillar, defining the 

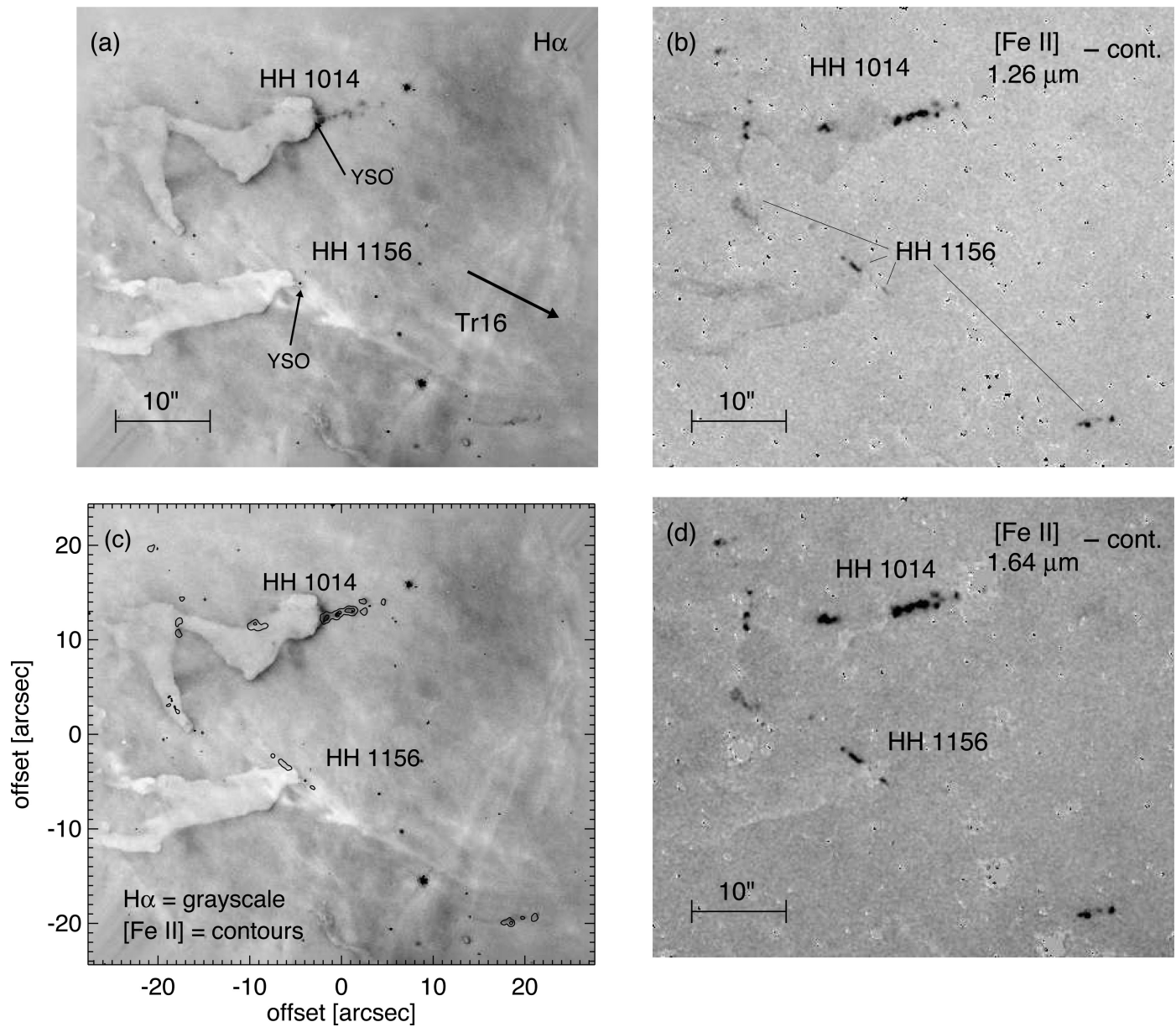

Figure 12. An $\mathrm{H} \alpha$ image (a) from Smith et al. (2010) shows HH 1014 emerging from the northern of two neighbouring dust pillars. New, continuum-subtracted [Fe II] images (b,d) reveal the HH 1014 counterjet. More surprising is the collimated, bipolar outflow that emerges from the head of the southern pillar, HH 1156, that appears to have no $\mathrm{H} \alpha$ counterpart (c).
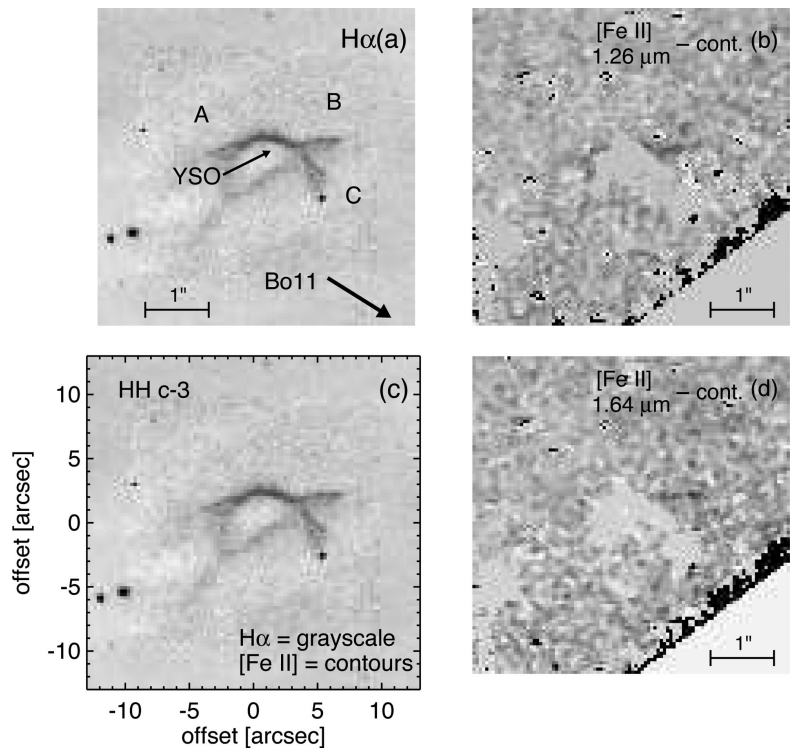

Figure 13. $\mathrm{HH} \mathrm{c}-3$ is a candidate jet, with extended $\mathrm{H} \alpha$ emission (a) possibly tracing a bipolar outflow. New, continuum-subtracted [Fe II] images $(b, c, d)$ trace collimated emission in feature B, although the poor signal to noise near the edge of the frame prohibits confident identification of the nature of this source. individual outflows that create the complicated shock structure surrounding the globule. Together with proper motion measurements of each feature (Reiter et al. in preparation), we can connect each feature with outflow phenomena. Therefore, these five candidate jets are assigned $\mathrm{HH}$ numbers, as described for each object individually below.

HH 1159 is a bow-shock-like knot that lies to the west of the extended globule (see Fig. 14) that was identified as HH c-4 by Smith et al. (2010). Bright [Fe II] emission traces the same C-shaped morphology as $\mathrm{H} \alpha$ and confirms that the cup of the $\mathrm{C}$ faces away from the globule (to the west). HH 1159 therefore resembles a bow shock moving towards the globule, as if launched by a source outside the globule. No extended [Fe II] emission reveals an accompanying jet body or otherwise indicates the origin of the feature.

HH 1160 (HH c-5 in Smith et al. 2010) emerges from the apex of the extended globule and appears to be a one-sided jet in $\mathrm{H} \alpha$ images (see Fig. 14). Like HH 1014, [Fe II] images reveal the counterjet inside the dusty globule. Together, the two limbs trace an $\sim 15$ arcsec long jet oriented at an $\sim 50^{\circ}$ angle compared to the major axis of the globule. The north-west and south-east sides of the jet appear to propagate away from a protostar that lies along the jet axis defined by [Fe II] (PCYC 787; Povich et al. 2011).

HH 1161 bursts from a curved surface protruding off the side of the extended globule. $\mathrm{H} \alpha$ images reveal a clear bipolar morphology tracing an $\sim 18$ arcsec long jet. However, $\mathrm{H} \alpha$ emission from the 

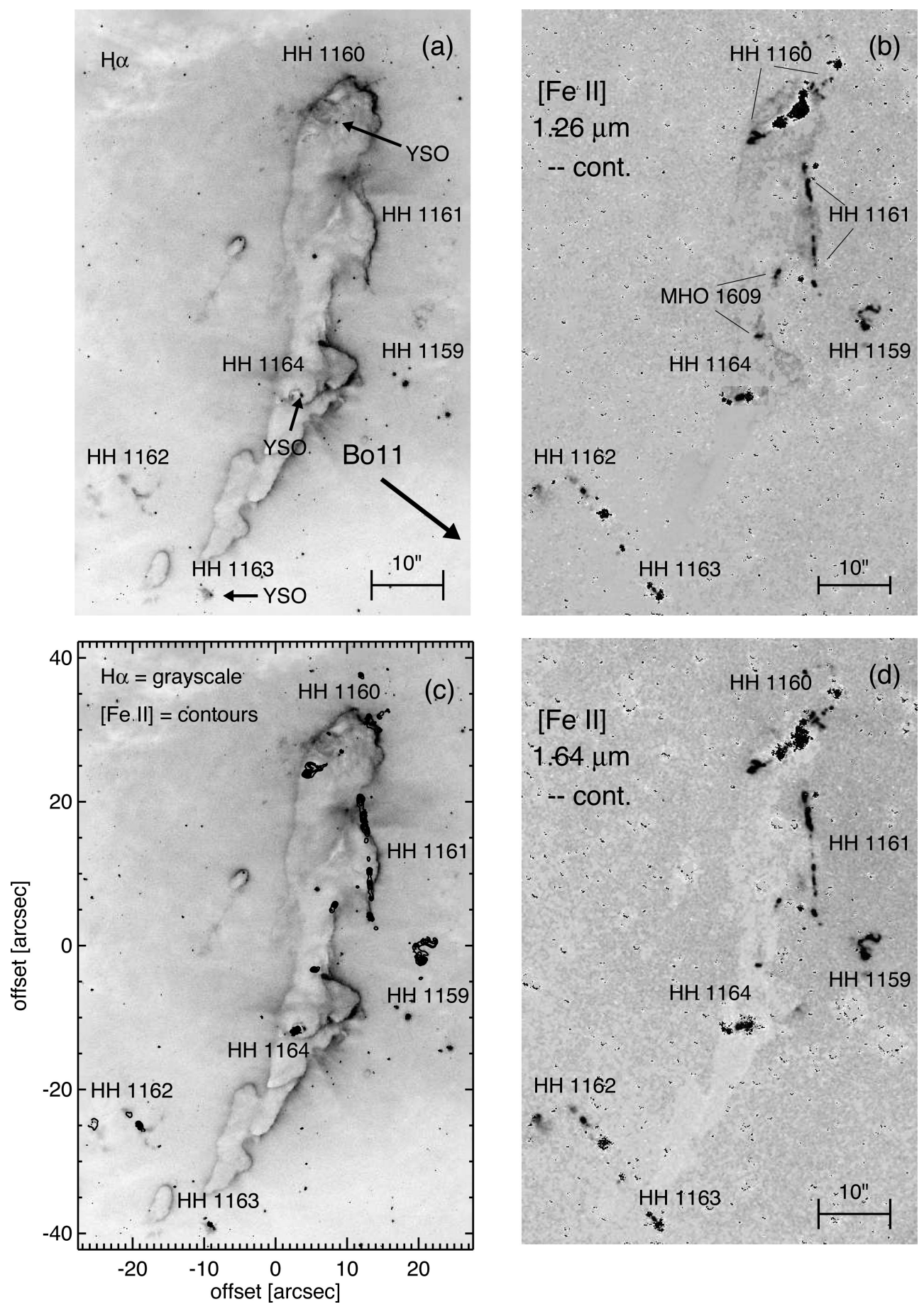

Figure 14. H $\alpha$ images (a) from Smith et al. (2010) and new near-IR [Fe II] images (b,c,d) of a globule in the south pillars with several candidate jets identified by Smith et al. (2010). [Fe II] observations and our forthcoming proper motion study confirm that these are bona fide HH objects. Features are labelled with their new HH numbers (see Section 3.2).

putative jet blends smoothly with the ionization front along the curved surface of the pillar, leading Smith et al. (2010) to identify it as candidate jet $\mathrm{HH}$ c-6.

Continuum-subtracted [Fe II] images clearly separate jet emission from the PDR, revealing a straight bipolar jet. The jet axis delineated by $[\mathrm{Fe} \mathrm{II}]$ emission does not appear to bend and is aligned nearly parallel to the major axis of the extended globule. In more distant portions of the jet, $\mathrm{H} \alpha$ and $[\mathrm{Fe}$ II] emission from the collimated jet coincide (see Fig. 14). Inside the globule, however, the [Fe II] jet remains straight, unlike the $\mathrm{H} \alpha$ emission which curves away from the jet axis, instead tracing the arc of the ionization front along the globule edge. No PCYC YSOs lie near HH 1161, although emission at $\lambda \geq 4.5 \mu \mathrm{m}$ suggests a deeply embedded protostar at the base of the southern limb of the jet.

HH 1162 (HH c-7 in Smith et al. 2010) lies on the opposite side of the globule from HH 1159, to the south-east of HH 1160 and HH 1161. Like HH 1159, HH 1162 appears to be a bow shock, located just off the edge of the globule. The curved morphology 
points to the south in this case, as though driven by a source located outside and possibly above the globule. Smith et al. (2010) posit that a star $\sim 30-40$ arcsec to the north-east may be the driving source.

[Fe II] emission traces the brightest $\mathrm{H} \alpha$ emission from $\mathrm{HH} 1162$. [Fe II] emission from $\mathrm{HH} 1162$ breaks the feature into two layers of [Fe II] knots with a single knot offset $\sim 4.5$ arcsec east of the chain of two knots. The three [Fe II] knots (see Fig. 14) do not trace the curve of the putative bow shock. No [Fe II] emission is seen to the north-east of HH 1162 nor near the candidate driving source identified by Smith et al. (2010).

$\mathrm{HH} 1163$ is an LL Ori-type object that lies immediately south of the extended globule that Smith et al. (2010) identified as $\mathrm{HH}$ c- 8 . A cone of $\mathrm{H} \alpha$ emission fans open to the east, with no obvious collimated jet body. Like the $\mathrm{H} \alpha$, [Fe II] emission from $\mathrm{HH} 1163$ extends $\sim 0.75$ arcsec to the east (see Fig. 14). Unlike $\mathrm{H} \alpha$, the stream of $[\mathrm{Fe} \mathrm{II}]$ emission is collimated, tracing the top edge of the cone of $\mathrm{H} \alpha$ emission. An additional [Fe $\mathrm{II}]$ knot lies $\sim 7$ arcsec to the north-east (see Fig. 14), along the jet axis defined by extended [Fe II] emission.

MHO 1609. A few bright knots stand out inside the globule in continuum-subtracted [Fe II] images. Two bright knots inside the narrow waist of the globule, just south of HH 1161, do not appear to be related to any of the HH objects identified by Smith et al. (2010). However, Hartigan et al. (2015) identified a molecular hydrogen outflow, MHO 1609, at this position in ground-based near-IR $\mathrm{H}_{2}$ images. The two [Fe II] knots seen in our WFC3-IR images appear to be at the same position as MHO 1609 knots ' $a$ ' and 'b'. Both [Fe II] knots clearly lie inside the ionization front tracing the edge of the globule, suggesting that these knots may be shock-excited knots in the embedded molecular jet.

$H H$ 1164. Further south in the globule, beneath MHO 1609, lies a loop of $\mathrm{H} \alpha$ emission emerging from a star in the globule. $\mathrm{H} \alpha$ emission on the right (west) side of the loop coincides with the position of a Spitzer-identified protostar (PCYC 790, see Table 3). Our new continuum-subtracted [Fe II] images reveal an $\sim 0.5$ arcsec long jet that extends to the east of the protostar. The [Fe II] jet extends through the middle of the $\mathrm{H} \alpha$ loop, tracing the familiar jet-cocoon morphology seen in, e.g. HH $666 \mathrm{O}$. We therefore assign this jet an HH number of 1164.

HH c-10. A series of curved shock structures that look like bow shocks in $\mathrm{H} \alpha$ are seen near the head of the same pillar that houses HH 903 (Smith et al. 2010). The implied outflow axis points to the south-west, towards $\mathrm{HH} 903$ which emerges from the middle of the pillar $\sim 90$ arcsec south of HH c-10. No emission from the putative $\mathrm{HH}$ c-10 bow shock is seen in [Fe II] emission (see Fig. 15). However, two IR bright point sources are detected at the head of the pillar. Two knots of [Fe II] emission emerge in the continuumsubtracted image and fall along the outflow axis implied by the $\mathrm{H} \alpha$ morphology. [Fe II] emission is brighter at $1.64 \mu \mathrm{m}$ than $1.26 \mu \mathrm{m}$, suggesting that this may be an embedded jet body. However, the bright point sources lead to imperfect continuum subtraction, so the true nature of $\mathrm{HH}$ c-10 remains unclear from images alone.

\subsection{Comparison of the $\mathrm{H} \alpha$ and [Fe II] intensity}

Fig. 16 shows comparisons between the $\mathrm{H} \alpha$ and $[\mathrm{Fe}$ II] emission in the $\mathrm{HH}$ jets with WFC3-IR observations. Both the jets and the ionization fronts along the pillar edges tend to be bright in $\mathrm{H} \alpha$. This is not the case in [Fe II], where emission is enhanced in the jets and may not be detected at all in the ionization front. To quantify this, we measure the [Fe $\mathrm{II}] 1.64 \mu \mathrm{m} / \mathrm{H} \alpha$ ratio and find that it
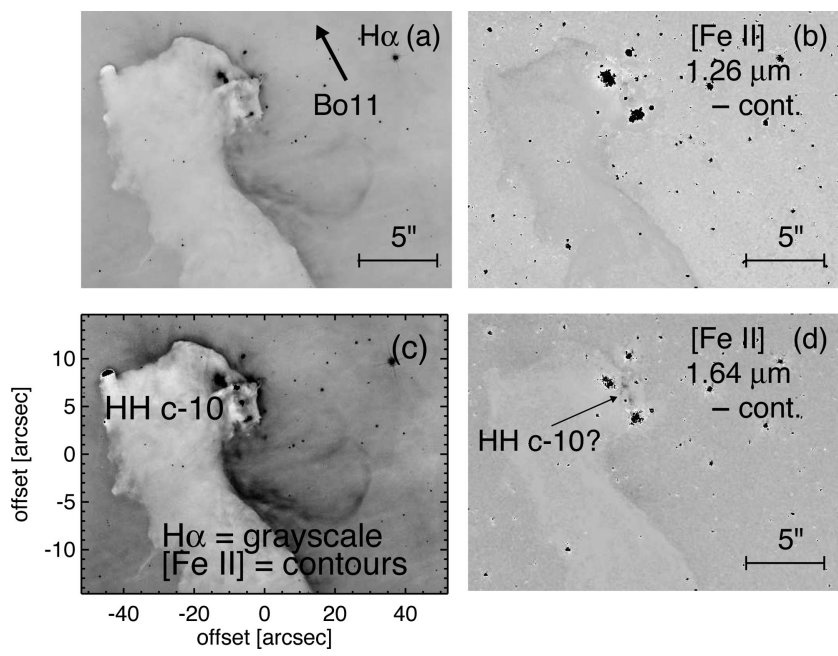

Figure 15. $\mathrm{HH}$ c-10 is a candidate jet identified as a bow shock in $\mathrm{H} \alpha$ images (a) by Smith et al. (2010). Faint [Fe II] $1.64 \mu \mathrm{m}$ emission (b,c,d) may trace the body of the corresponding jet that emerges from the pillar head.

is $\gtrsim 5$ times higher in the jet than the adjacent ionization front (see Fig. 16).

We also compare the $\mathrm{H} \alpha$ intensities measured by Smith et al. (2010) to the intensity of both [Fe II] lines and their ratio over roughly the same portion of the jet. Neither quantity is correlated with the intensity of the $\mathrm{H} \alpha$ emission. However, as shown by Reiter $\&$ Smith (2013), $\mathrm{H} \alpha$ and [Fe II] trace different material in the jet and are often bright in different places along the length of the jet. $\mathrm{H} \alpha$ traces the density of material in the ionized outer radii of the outflow while $[\mathrm{Fe} \mathrm{II}]$ traces the density of the jet core. These densities are not necessarily correlated with each other or the strength of the incident ionizing flux (see Fig. 17). In addition, the $\mathrm{H} \alpha$ intensity scales as $n_{\mathrm{e}}^{2}$, so relatively small offsets in density will enhance the spread in jet intensities.

\section{$3.4[\mathrm{Fe} \mathrm{II}]$ ratio tracings}

The [Fe II] transitions we observed with HST are the two brightest lines that originate from the $\mathrm{a}^{4} \mathrm{D}$ level. The intrinsic flux ratio, $\mathcal{R}=\lambda 12657 / \lambda 16437$, will therefore be determined by atomic physics. In the absence of any reddening, the $1.26 \mu \mathrm{m}$ line will be brighter than the $1.64 \mu \mathrm{m}$ line. However, the intrinsic flux ratio $\mathcal{R}$ is only known as well as the transition probabilities, which remain uncertain (e.g. Nussbaumer \& Storey 1988; Quinet 1996; Bautista \& Pradhan 1998; Rodríguez-Ardila et al. 2004; Smith \& Hartigan 2006; Deb \& Hibbert 2010; Bautista et al. 2013, 2015). Observational estimates of the intrinsic flux ratio $\mathcal{R}$ have yielded values from $\mathcal{R}=1.11$ in HH 1 (Giannini et al. 2015) to $\mathcal{R}=1.49$ in P Cygni (Smith \& Hartigan 2006). Lower values for the intrinsic ratio $\mathcal{R}$ correspond to less extinction for the same measured ratio (see discussion in Reiter et al. 2015b).

We measure the ratio $\mathcal{R}$ along the length of the jet in new, continuum-subtracted [Fe II] images (see e.g. Fig. 18; ratio tracings for the other jets in the sample are available in the online supplement). For each jet, we indicate the regions of the jet considered on the $[\mathrm{Fe}$ II] $1.64 \mu \mathrm{m}$ image. To obtain a representative value for each jet, we take the ratio of a bright knot in roughly the same portion of the jet that Smith et al. (2010) extracted in $\mathrm{H} \alpha$, although this is not possible in all cases (e.g. HH 1005 and HH 1007). We have not 

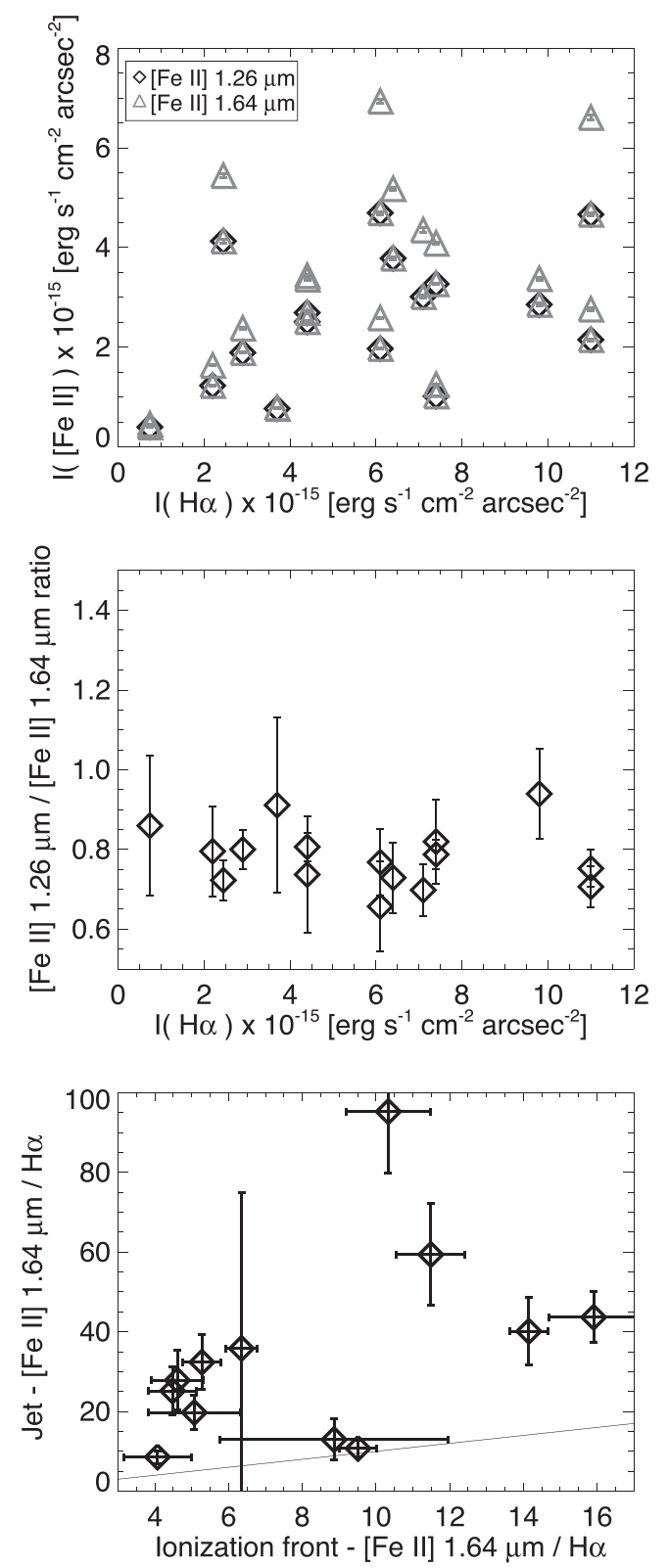

Figure 16. Top: [Fe II] $1.26 \mu \mathrm{m}$ (black diamonds) and $1.64 \mu \mathrm{m}$ (grey triangles) jet intensity plotted against the $\mathrm{H} \alpha$ intensity measured for a similar portion of that jet by Smith et al. (2010, see table 4). Error bars are smaller than the plot symbols. Middle: $[\mathrm{Fe}$ II] ratio $\mathcal{R}$ measured in a bright portion of the jet plotted as a function of the $\mathrm{H} \alpha$. Bottom: comparison of the flux ratio [Fe II] $1.64 \mu \mathrm{m} / \mathrm{H} \alpha$ measured in the jet compared to the adjacent ionization front for jets embedded in a globule. The solid line shows where the two are equal.

corrected for line-of-sight reddening towards Carina (Smith 1987, 2002).

Measuring the $[\mathrm{Fe} \mathrm{II}]$ ratio in images is most illustrative of how the reddening changes along the length of the jet. Unlike spectra, narrow-band images may be contaminated with other emission lines that fall within the filter bandwidth. Unequal widths of the narrowband filters may result in different amounts of contamination from other emission lines in the images (i.e. $15.2 \mathrm{~nm}$ for $F 126 \mathrm{~N}$ compared to $20.9 \mathrm{~nm}$ for $F 164 N$ ). For example, Br12 and Br11 fall in the $F 164 N$ and $F 167 N$ filters, respectively. He I $1.253 \mu \mathrm{m}$ may contaminate images obtained with the $F 126 N$ filter; indeed, this line

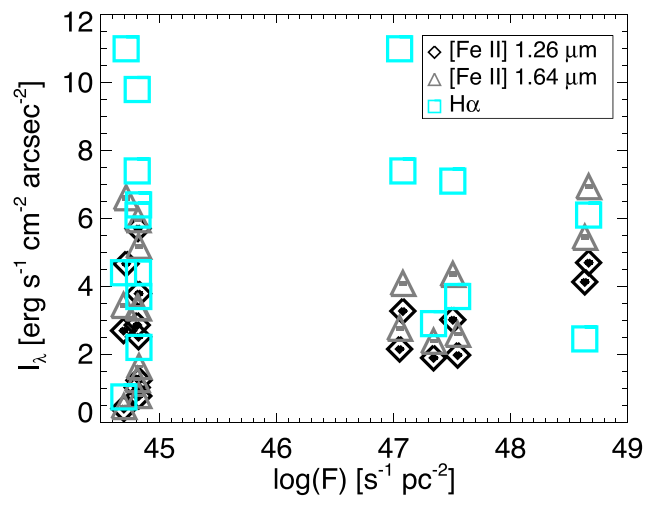

Figure 17. Representative jet intensity in [Fe II] $1.26 \mu \mathrm{m}$ (black diamonds), $1.64 \mu \mathrm{m}$ (grey triangles), and $\mathrm{H} \alpha$ (from Smith et al. 2010, cyan squares) plotted versus the incident ionizing photon flux (listed in Table 2).

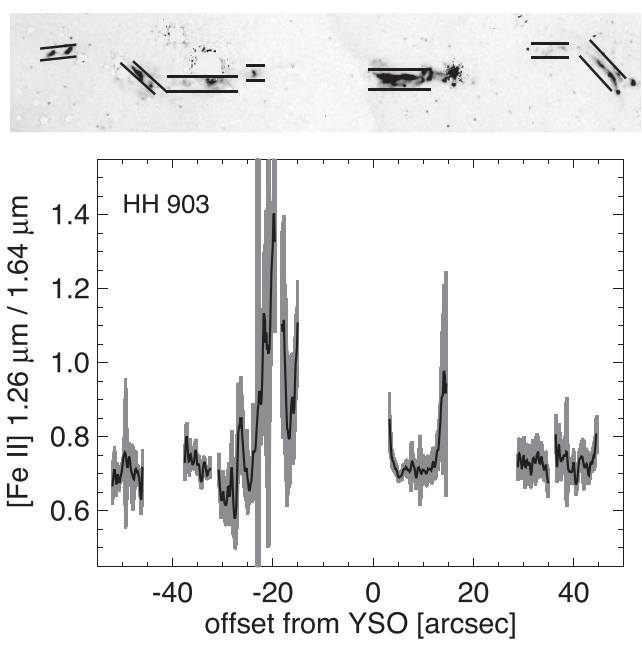

Figure 18. Top: [Fe II] $1.64 \mu \mathrm{m}$ image of $\mathrm{HH} 903$ with lines that show the area extracted to measure the [Fe II] ratio along each portion of the jet. Bright stars in the field have been masked. Bottom: tracing of the [Fe II] ratio $\mathcal{R}=\lambda 12657 / \lambda 16437$ along HH 903 . The grey shaded area surrounding the line shows the $1 \sigma$ uncertainty in the ratio at that point along the jet. Ratio tracings for the other jets presented in this work are in the supplementary material available online.

is observed to be brighter than theoretical expectation in the Orion bar and Orion S regions (see e.g. Luhman, Engelbracht \& Luhman 1998).

Regardless of potential contamination in the absolute [Fe II] line ratio, images allow us to trace the relative reddening as a function of position along the jet. All of the jets have ratios that remain below the intrinsic value along the length of the jet, often below $\mathcal{R} \approx 1$ (see Fig. 19) corresponding to $\mathrm{A}_{V} \sim 5 \mathrm{mag}$ for $\mathcal{R}=1.49$ (see fig. 9 in Reiter \& Smith 2013), or $\mathrm{A}_{V} \sim 1-2 \mathrm{mag}$ for $\mathcal{R}=1.11-1.20$ (Giannini et al. 2015). In the $\mathrm{H}$ II region, the ratio stays near a constant value, similar to the steady $\mathcal{R}$ values found by Reiter et al. (2015b) in HH 666. Reiter \& Smith (2013) used the change in $\mathcal{R}$ in HH 1066 to argue for an optically thick flared circumstellar disc increasing the reddening near the jet-driving source. A similar circumstellar geometry may explain the dip in $\mathcal{R}$ near the position of the driving source in HH 1006 and HH 1156. 


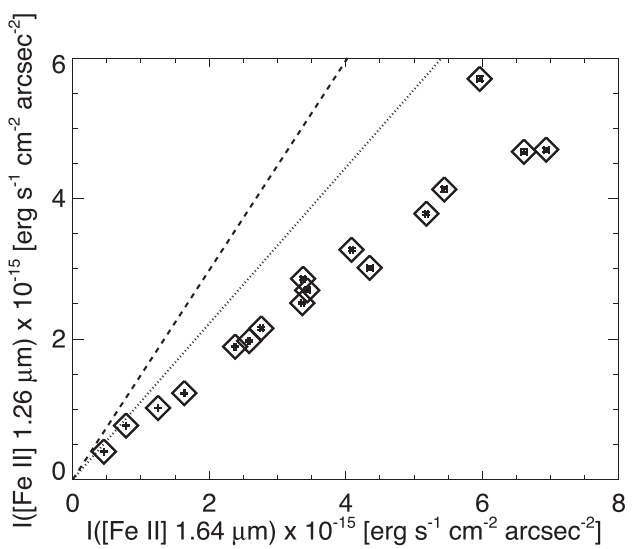

Figure 19. Intensity of [Fe II] $1.26 \mu \mathrm{m}$ plotted as a function of the [Fe II] $1.64 \mu \mathrm{m}$ intensity. Lines corresponding to the expected line fluxes for zero reddening for an intrinsic flux ratio $\mathcal{R}=1.49$ (Smith \& Hartigan 2006, dashed line) and $\mathcal{R}=1.11$ (Giannini et al. 2015, dotted line) are overplotted.

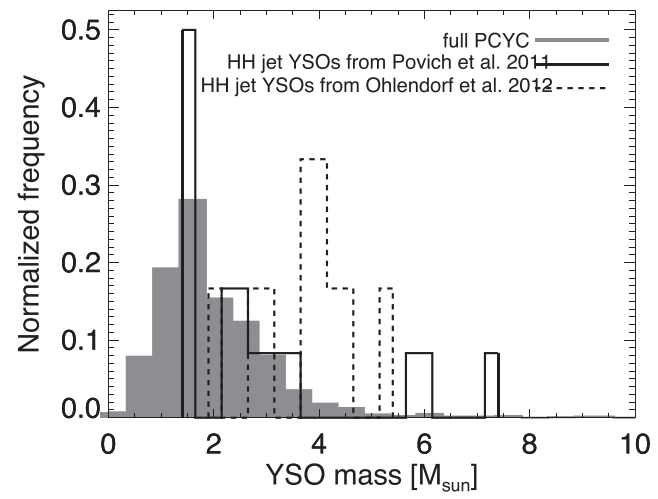

Figure 20. A normalized histogram of the masses of all PCYC protostars with model fits to the IR SED from Povich et al. (2011) is shown in grey. The overplotted solid line histogram shows the distribution of YSO masses of the jet-driving sources we identified from the PCYC. The dashed-line histogram shows the jet-driving protostar masses determined by Ohlendorf et al. (2012).

\subsection{Candidate driving sources}

For this programme, we targeted HH jets in the Carina Nebula with a candidate protostar identified along the jet axis. [Fe II] emission from most jets extends inside the dust pillar, clearly connecting the larger outflow seen in $\mathrm{H} \alpha$ to the embedded IR YSO. We list the PCYC number of the matched driving sources and the physical properties estimated from model fits to the IR SED from Povich et al. (2011) in Table 3. Ohlendorf et al. (2012) also identified candidate driving sources for some of the HH jets in Carina discovered by Smith et al. (2010). Their YSO identifications and model fits are also included in Table 3. For the four sources where the identified driving source has been modelled by both Povich et al. (2011) and Ohlendorf et al. (2012), the derived source properties agree to within a factor of 2 , and often to within a few per cent. For the remainder of this analysis, we will focus on the model results obtained by Povich et al. (2011).

Fig. 20 compares the masses of the jet-driving protostars in Carina to all the YSOs in Carina modelled by Povich et al. (2011). One of the goals of the HST survey for [Fe II] emission from the $\mathrm{HH}$ jets in Carina is to test the physical properties of the jets driven by protostars sampling the intermediate-mass $\left(\sim 2-8 \mathrm{M}_{\odot}\right)$ range. Estimated driving source masses range from 1.4-7.5 $\mathrm{M}_{\odot}$. The SEDs of the jet-driving sources are consistent with young YSOs (although 4/12 have an ambiguous evolutionary classification).

For five jets in this sample, the driving source remains somewhat ambiguous or unknown. In the case of $\mathrm{HH} 1005$, point-like IR emission consistent with being a YSO has been identified near the outflow axis (see Ohlendorf et al. 2012). However, [Fe II] emission from the jet seen inside the natal dust pillar does not clearly connect the larger scale $\mathrm{H} \alpha$ outflow to any of the nearby candidate protostellar objects. Most of the remaining outflows - HH 900, HH 901, $\mathrm{HH} 902$ - lie close to the brightest emission from the $\mathrm{H}$ II region, where the bright and variable background will limit sensitivity to point source emission (see discussion in Povich et al. 2011). No $[\mathrm{Fe}$ II $]$ emission is detected inside the globule from either $\mathrm{HH} 900$ or HH 901, rather it begins $\sim 1$ arcsec away from the edge of the globule. Reiter \& Smith (2013) and Reiter et al. (2015a) have explored the possibility that feedback from nearby massive stars may have compressed the globules, leading to high densities that obscure the jet-driving protostar and shield the inner jet. However, some jets without a detected driving source lie far from $\operatorname{Tr} 14$ and $\operatorname{Tr} 16$. HH 1161 emerges from a globule deeper in the south pillars. While [Fe II] emission from HH 1161 does reach inside the globule (unlike $\mathrm{HH} 900$ and HH 901), no protostar has been detected near the jet axis.

Jet dynamical ages may be estimated by assuming that a jet knot reached its current position by travelling at a constant velocity. This provides an independent estimate of the age and therefore evolutionary stage of the driving source. Individual jet velocities have only been measured for $5 / 19$ jets in this work. Adopting the median knot velocity found by Reiter \& Smith (2014) for four $\mathrm{HH}$ jets in Carina $-v_{j e t}=140 \mathrm{~km} \mathrm{~s}^{-1}-$ we find that the median dynamical age of the $\mathrm{HH}$ jets presented in this study is $\sim 3500 \mathrm{yr}$. This age range is very uncertain, but in any case is only a few per cent of the Class 0 and Class I lifetimes estimated for low-mass sources (0.16 Myr and 0.54 Myr, respectively; Evans et al. 2009).

\section{DISCUSSION}

We detect $\left[\mathrm{Fe}_{\mathrm{II}}\right]$ emission in all of the $\mathrm{HH}$ jets in the Carina Nebula targeted for follow-up observation with WFC3-IR. In addition, three candidate jets also fell within the area imaged with WFC3-IR. Of the three, only HH 1156 (formerly HH c-14) has a clear bipolar jet morphology. $\mathrm{HH} \mathrm{c}-3$ and $\mathrm{HH} \mathrm{c-10}$ also show faint [Fe II] emission, but their true nature remains unclear. Altogether new, continuumsubtracted [Fe II] images combined with archival images of four other sources yield a sample of $21 \mathrm{HH}$ objects corresponding to at least 18 individual jets (see Table 2).

\subsection{Strong [Fe II] emission from externally irradiated $\mathrm{HH}$ jets}

Bright $[\mathrm{Fe}$ II] emission traces a collimated jet in almost every source presented in this paper. Unlike $\mathrm{H} \alpha$ images, where jet emission is often confused with the pillar edge or surrounding cocoon, continuum-subtracted [Fe $\mathrm{FI}$ images make it easy to distinguish jet emission from the PDR (see Section 3.3 and Fig. 21). For sources with a clear jet-like morphology, the detection of [Fe II] emission demonstrates that the jet is not completely ionized, but instead maintains a neutral core. Therefore, the mass-loss rates estimated by Smith et al. (2010) are lower limits to the true mass-loss rate in the jet. We revise jet mass-loss rates to account for this in Section 4.4

As pointed out by Bally et al. (2006), to first order, the $\mathrm{H} \alpha$ intensity of a jet with a neutral core will be proportional to the local 
Table 2. Jet properties.

\begin{tabular}{|c|c|c|c|c|c|c|c|c|c|}
\hline Name & $\begin{array}{l}L_{\mathrm{tot}}{ }^{a} \\
(\operatorname{arcsec})\end{array}$ & $\begin{array}{l}L_{\text {inner }}^{a} \\
(\operatorname{arcsec})\end{array}$ & $\begin{array}{l}L_{1} \\
(\mathrm{pc})\end{array}$ & $\begin{array}{l}W_{\text {pillar }} \\
(\operatorname{arcsec})\end{array}$ & $\begin{array}{l}\text { Ionizing } \\
\text { cluster }\end{array}$ & $\begin{array}{l}\text { Proj. dist. } \\
\text { (pc) }\end{array}$ & $\begin{array}{c}\dot{M}_{\text {jet }} \\
\left(\mathrm{M}_{\odot} \mathrm{yr}^{-1}\right)\end{array}$ & $\underset{c}{\Delta \dot{M}}$ & Comment \\
\hline HH 666 & 308 & 16.5 & 0.18 & 34 & $\operatorname{Tr} 16$ & 14.2 & $1.2 \mathrm{e}-5$ & 51 & See also Reiter \& Smith (2013). \\
\hline HH 901 & 20 & 3.5 & 0.04 & 2 & $\operatorname{Tr} 14$ & 2.0 & $4.7 e-6$ & 42 & See also Reiter \& Smith (2013). \\
\hline HH 902 & 35 & 8 & 0.09 & 11 & $\operatorname{Tr} 14$ & 2.1 & $1.0 \mathrm{e}-5$ & 57 & See also Reiter \& Smith (2013). \\
\hline НH 1066 & 7 & 2 & 0.02 & 1.25 & $\operatorname{Tr} 14$ & 2.9 & $1.7 \mathrm{e}-6$ & 12 & See also Reiter \& Smith (2013). \\
\hline HH 900 & 46 & 10 & 0.11 & 2.35 & $\operatorname{Tr} 16$ & 7.9 & $1.7 \mathrm{e}-5$ & 30 & See also Reiter et al. (2015a). \\
\hline HH 903 & 167 & 17 & 0.19 & 28 & Bo11 & 13.0 & $1.0 \mathrm{e}-6$ & 6 & \\
\hline НH 1004 & 27 & 9.6 & 0.11 & 14 & Bo11 & 5.7 & $1.2 \mathrm{e}-6$ & 10 & \\
\hline HH 1005 & 71 & 10 & 0.11 & 33 & Bo11 & 5.9 & $1.2 \mathrm{e}-6$ & 10 & \\
\hline HH 1006 & 67.5 & 6 & 0.07 & 6 & Bo11 & 7.1 & $3.0 \mathrm{e}-6$ & 126 & \\
\hline $\mathrm{HH} 1007^{d}$ & - & - & - & - & Bo11 & 29.9 & - & - & No extended jet component. \\
\hline HH 1010 & 48 & 7 & 8.5 & 0.08 & $\operatorname{Tr} 16$ & 29.1 & $2.4 \mathrm{e}-6$ & 35 & \\
\hline HH 1014 & 22 & 6 & 7 & 0.07 & $\operatorname{Tr} 16$ & 11.4 & $3.9 \mathrm{e}-6$ & 89 & \\
\hline $\mathrm{HH} 1015^{d}$ & $6-18$ & 6 & 6 & 0.07 & Bo11 & 30.0 & $1.4 \mathrm{e}-7$ & 17 & \\
\hline $\mathrm{HH} \mathrm{c-3}$ & - & 2.5 & 2.5 & 0.03 & Bo11 & 21.8 & $8.4 \mathrm{e}-8$ & 6 & Only component B from Smith et al. (2010). \\
\hline НH 1159 & - & - & - & - & Bo11 & 21.2 & - & - & No extended jet component. \\
\hline НH 1160 & 15.5 & 6 & 7 & 0.07 & Bo11 & 21.1 & $2.1 \mathrm{e}-7$ & 17 & \\
\hline HН 1161 & 18.6 & 6 & 7 & 0.07 & Bo11 & 21.1 & $2.1 \mathrm{e}-7$ & 5 & \\
\hline HH 1162 & - & - & - & - & Bo11 & 20.3 & - & - & No extended jet component. \\
\hline НH 1163 & - & 2 & - & 0.02 & Bo11 & 20.5 & $7.2 \mathrm{e}-8$ & - & \\
\hline НH 1164 & 1.7 & 1.7 & 9.25 & 0.02 & Bo11 & 20.8 & $6.0 \mathrm{e}-8$ & - & Not detected by Smith et al. (2010). \\
\hline $\mathrm{HH} \mathrm{c-10}$ & - & - & - & - & Bo11 & 12.9 & - & - & No extended jet component. \\
\hline HH 1156 & 44.5 & 2 & 1 & 0.02 & $\operatorname{Tr} 16$ & 11.5 & $1.4 \mathrm{e}-6$ & - & Jet body not detected by Smith et al. (2010). \\
\hline
\end{tabular}

Lyman continuum flux. We might expect weaker $\mathrm{H} \alpha$ emission from jets like HH 1015 that lie far from their ionizing sources. Both $\mathrm{H} \alpha$ and $[\mathrm{Fe} \mathrm{II}]$ emission are weak in $\mathrm{HH} 1015$, suggesting less external excitation of the jet. In general, however, the observed strength of the $[\mathrm{Fe}$ II] emission does not correlate with the $\mathrm{H} \alpha$ intensity of the jet (see Section 3.3 and Fig. 16) and does not depend on environment. [Fe II] is often a better tracer of the fast, collimated jet than $\mathrm{H} \alpha$ (see Section 4.2 and Reiter et al. 2015a,b). This is true inside the dust pillars where only $[\mathrm{Fe} \mathrm{II}]$ traces the jet and outside the pillar where both $\mathrm{H} \alpha$ and $[\mathrm{Fe}$ II] are visible. Bright $\mathrm{H} \alpha$ emission from ionized gas in the $\mathrm{H}_{\text {II }}$ region may obscure fainter jet emission, especially in the complicated environment near $\eta$ Carinae. In contrast, bright [Fe II] emission is confined to the core of protostellar jets where densities are sufficient to shield the $\mathrm{Fe}^{+}$from further ionization. Confusion with the environment may explain why the body of $\mathrm{HH}$ 1156 cannot be identified in $\mathrm{H} \alpha$ even though bright [Fe II] emission clearly traces the bipolar jet.

Strong $[\mathrm{Fe}$ II] jet emission is observed from deeply embedded, and therefore undetected, protostars (e.g. HH 900 and HH 1161, see Figs 6 and 14, respectively) as well as those readily apparent in $\mathrm{H} \alpha$ images (e.g. HH 1163, see Fig. 14). If material entrained from the environment was solely responsible for shielding $\mathrm{Fe}^{+}$from further ionization, strong [ $\mathrm{Fe} \mathrm{II}]$ emission in jets would persist only inside the dusty pillars. This cannot explain bright [Fe II] emission from jet limbs that emerge from protostars located near pillar edges (e.g. HH 903, HH 1004, and HH 1006, see Figs 7, 8, and 9, respectively), nor can it explain strong [ $\mathrm{Fe}$ II] emission in jets driven by unobscured protostars (e.g. HH 1163, see Fig. 14). Instead, the ubiquity of strong [Fe II] emission from the $\mathrm{HH}$ jets in Carina suggests that dust in the jet must be introduced locally, either launched into the jet from the disc or created in the outflow.

\subsection{Two-component jets}

Reiter \& Smith (2013) showed that [Fe II] emission traces neutral material in dense jets. In some cases (e.g. HH 901), the ionization front in the jet can be spatially resolved. Bright [Fe II] emission in the jet peaks behind the $\mathrm{H} \alpha$, tracing neutral material located behind the ionized skin of the jet (and further away from the ionizing source). For other jets in Carina, $\mathrm{H} \alpha$ and near-IR [Fe II] emission appear to trace two distinct outflow components. In both $\mathrm{HH} 666$ and $\mathrm{HH}$ 900, a slower, wider-angle cocoon of $\mathrm{H} \alpha$ emission surrounds the fast, highly collimated jet seen in [Fe II] (Reiter et al. 2015a,b).

Four jets presented in this paper also show this two-component, jet-outflow morphology. HH 1004 (SW), HH 1161, HH 1164, and $\mathrm{HH} 1066$ all show $\mathrm{H} \alpha$ emission that is parallel to, but offset from, the $[\mathrm{Fe}$ II] jet (see Figs 8, 14, and 5, respectively). Both HH 1161 and HH 1164 were originally identified as candidate jets by Smith et al. (2010) because $\mathrm{H} \alpha$ emission from the jet is confused with the ionization front along the surface of the globule. New [Fe II] images reveal steady, collimated jets with morphologies that converge with $\mathrm{H} \alpha$ only near the terminus of the continuous inner jet, similar to HH 666 M and HH 900 (see Fig. 6 and Reiter et al. 2015a).

HH 903, HH 1004 (NE), and HH 1014 disrupt the smooth morphology of the ionization front as they break out of the pillar. Broad $\mathrm{H} \alpha$ emission that extends into the $\mathrm{H}$ in region along the body of the jet may trace entrained material that is being dragged out of the pillar. Unlike the $\mathrm{H} \alpha$ sheaths seen in $\mathrm{HH} 1004$ (SW) and HH 666, these broad $\mathrm{H} \alpha$ components survive for only a short length. $\mathrm{H} \alpha$ emission from more distant portions of the jet are more collimated, and well matched to the jet morphology seen in [Fe II]. This is similar to $\mathrm{HH}$ $666 \mathrm{M}$ where $\mathrm{H} \alpha$ traces the wider-angle outflow inside the pillar, and the ionized skin of the bare jet in the $\mathrm{H}$ II region. 


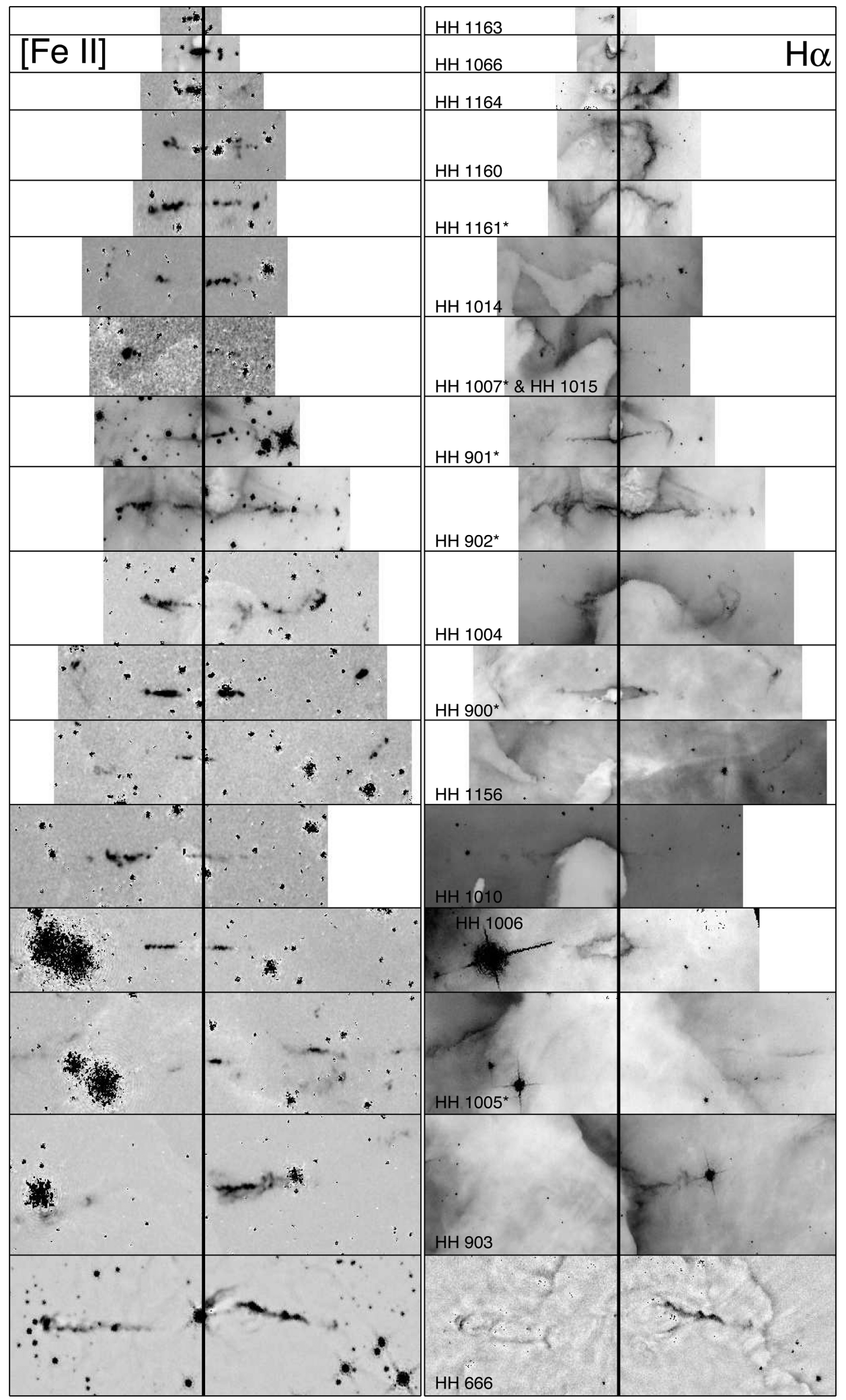

Figure 21. A comparison of $17 \mathrm{HH}$ jets in the Carina Nebula that have been observed in [Fe II] with WFC3-IR (HH c-3 is not shown). Jets are shown on the same spatial scale, centred at the driving source position. For jets without a detection of the driving source (indicated with an asterisk next to the name) the YSO location is estimated based on the morphology of the jet emission. 
Each of these two-component jets emerges from an embedded protostar. Deeply embedded sources like HH 900 and HH 1161, where no protostar has been identified, only show two components beyond the edge of the globule. When the protostar can be identified in $\mathrm{H} \alpha$ images, as in $\mathrm{HH} 666$ and $\mathrm{HH}$ 1164, both $\mathrm{H} \alpha$ and [Fe II] trace the outflow from the source. HH 1163 illustrates a third situation, where a two-component system is observed from a protostar that is not embedded in a pillar. However, Smith et al. (2010) note that $\mathrm{HH}$ 1163 resembles an LL Ori object, suggesting that wide-angle $\mathrm{H} \alpha$ emission may come from interaction with a side wind, rather than being entrained by the jet.

The only other jet seen emerging from an exposed protostar, $\mathrm{HH}$ 1156, also has a strong, bright, collimated [Fe II] jet. However, only the bow shock identified by Smith et al. (2010) shows any $\mathrm{H} \alpha$ emission. The immediate vicinity of $\mathrm{HH} 1156$ is dark in $\mathrm{H} \alpha$ images, hinting at uneven foreground extinction that may obscure visual wavelength emission from the jet. However, the line-of-sight reddening, estimated from the $\left[\mathrm{Fe}_{\mathrm{II}}\right]$ ratio $\mathcal{R}$, does not vary significantly between the inner jet and the shock that can be seen in $\mathrm{H} \alpha$. It remains puzzling that the protostar can be seen at visual wavelengths, but no part of the inner jet can be identified in $\mathrm{H} \alpha$ images.

\subsection{Knot structure}

New [Fe II] images trace portions of the $\mathrm{HH}$ jets in Carina not seen in $\mathrm{H} \alpha$. The morphology of the [Fe $\mathrm{II}$ emission in some $\mathrm{HH}$ objects suggest that these separate knots may be part of a larger coherent jet. For example, HH 1007 lies along the outflow axis defined by $\mathrm{HH} 1015$, and appears to be the counterjet bow shock. Velocity measurements are required to determine how these knots relate to individual coherent jet structures.

Smooth, continuous emission from inner jets (suggesting no strong shocks) hint at a sustained accretion outburst that powers the jet. The median inner jet length is $\sim 6$ arcsec, corresponding to an outburst duration of $475 \mathrm{yr}$ (assuming $v_{\text {jet }}=140 \mathrm{~km} \mathrm{~s}^{-1}$ for all jets). Only the shortest jets are consistent with the observed duration of FU Orionis outbursts (Hartmann \& Kenyon 1996). It is unclear how the typical decay time of an accretion burst from an intermediate-mass star compares to an FU Orionis outburst, as accretion outbursts have only been detected in a few intermediatemass sources (e.g. Hinkley et al. 2013).

An alternate way to estimate how the duration of an accretion outburst scales with protostellar mass is to consider the viscous evolution. Viscosity determines the structure and evolution of an accretion disc. Therefore, the outburst duration will be governed by the viscous time-scale,

$t_{\mathrm{vis}} \sim \frac{R_{\mathrm{out}}^{2}}{v}$,

where $R_{\text {out }}$ is the outermost radius in the disc that is unstable to accretion and $v$ is the viscosity. The viscosity itself is a function of the local sound speed $c_{\mathrm{s}}$, and the Keplerian angular velocity $\Omega_{\mathrm{K}}=\sqrt{\frac{G M_{\star}}{R_{\text {out }}^{3}}}$ at radius $R_{\text {out }}$ for a star of mass $M_{\star}$ giving

$v=\frac{\alpha c_{\mathrm{s}}^{2}}{\Omega_{\mathrm{K}}}$,

where $\alpha$ is the viscosity parameter (Shakura \& Sunyaev 1973). If we assume that $\alpha$ and $c_{\mathrm{S}}$ are the same in protostellar accretion discs, regardless of the spectral type of the central star, then we can estimate the mass-dependence of the viscous time as

$t_{\text {vis }} \sim R_{\text {out }}^{2} \sqrt{\frac{G M_{\star}}{R_{\text {out }}^{3}}} \propto R_{\text {out }}^{1 / 2} M_{\star}^{1 / 2}$.

Thus, the viscous time for a $M_{\star}=10 \mathrm{M}_{\odot}$ object will be $\sim 3 \times$ longer than for a $1 \mathrm{M}_{\odot}$ star at the same $R_{\text {out }}$.

Magnetorotational instability (MRI; Balbus \& Hawley 1991) is the most promising source of viscosity in protostellar discs. Only in portions of the disc where the gas is coupled to the magnetic field will undergo MRI and be unstable to accretion. If the spectral type of the star meaningfully changes the radius in the disc where this occurs, then the outermost radius that is unstable to accretion, $R_{\text {out }}$, might be larger for higher mass stars, leading to longer outbursts. This estimate is clearly too simplistic to reflect the complicated physics of real discs (e.g. non-zero viscosity in the 'dead zone' of the disk, Bae et al. 2013) but nevertheless show that longer accretion outbursts are plausible in intermediate-mass protostars.

\subsection{Revised mass-loss rate estimates}

Smith et al. (2010) estimated the mass-loss rate of the HH jets in Carina from the H $\alpha$ EM. Reiter \& Smith (2013) showed that for a jet with a low-ionization (neutral) core that is not traced by $\mathrm{H} \alpha$, this may underestimate the mass-loss rate by as much as an order of magnitude. Following Bally et al. (2006) and Reiter \& Smith (2013), we compute new mass-loss rate estimates that include the neutral jet core in two ways. First, we estimate the minimum density, $n_{\min }$, required to maintain a slow-moving ionization front in the jet. Secondly, we estimate the mass-loss rate in the neutral jet by comparing it to the photoablation rate, using the known ionizing photon luminosity from the O-type stars in the Carina Nebula (Smith 2006).

The minimum density required for a jet to remain optically thick to Lyman continuum radiation is

$n_{\min }=\sqrt{\frac{Q_{\mathrm{H}}}{4 \pi D^{2}} \frac{\sin (\beta)}{2 \alpha_{\mathrm{B}} r}}$

where $Q_{\mathrm{H}}$ is the ionizing photon luminosity incident on the jet at an angle $\beta$ from a distance $D$, and $\alpha_{\mathrm{B}} \approx 2.6 \times 10^{-13} \mathrm{~cm}^{3} \mathrm{~s}^{-1}$ is the case $\mathrm{B}$ recombination coefficient for hydrogen. We have assumed a cylindrical jet column with radius $r$. By requiring that the density of the neutral jet, $n_{\mathrm{H}}$, is at least as large as this minimum density $\left(n_{\mathrm{H}} \geq n_{\min }\right)$, we can derive a lower limit on the mass-loss rate. For a cylindrical jet, the mass-loss rate is

$\dot{M}=n_{\min }\left|\pi \mu m_{\mathrm{H}} v_{\mathrm{jet}} r^{2}\right|$,

where $\mu$ is the mean molecular weight $(\approx 1.35), m_{\mathrm{H}}$ is the mass of hydrogen, and $v_{\text {jet }}$ is the jet velocity. Velocities have only been measured for a few of the HH jets in Carina, so we assume the median knot velocity measured by Reiter \& Smith (2014), $v_{\text {jet }}=$ $140 \mathrm{~km} \mathrm{~s}^{-1}$, for the remaining jets. We find a median mass-loss rate of $\sim 9 \times 10^{-8} \mathrm{M}_{\odot} \mathrm{yr}^{-1}$, similar to the median mass-loss rate estimated from the $\mathrm{H} \alpha \mathrm{EM}, \sim 1 \times 10^{-7} \mathrm{M}_{\odot} \mathrm{yr}^{-1}$ (Smith et al. 2010). Comparing these two estimates suggests that most of the $\mathrm{HH}$ jets in Carina support densities high enough to shield a neutral jet core, and indeed, we detect [Fe II] emission in every jet targeted with this sample.

Both of these estimates provide a lower limit on the jet mass-loss rate. To further improve the estimated mass-loss rate, we can use the length of the inner jet as a measure of how long the cylinder of 
ejected mass survives photoablation in the $\mathrm{H}_{\mathrm{II}}$ region. For a neutral jet core to persist, the jet density must be $n_{\mathrm{H}} \geq n_{\min }$ throughout the length of the continuous inner jet. Therefore, for a jet in an $\mathrm{H}$ II region to remain visible out to a distance $L_{1}$ from the driving source, it must have a mass-loss rate of at least $\dot{M}_{\text {jet }} \geq L_{1} \dot{m}$ where $\dot{m}$ is the photoablation rate of the jet in the $\mathrm{H}$ II region (Bally et al. 2006). For a cylindrical jet, this corresponds to a mass-loss rate

$\dot{M} \approx \frac{L_{1} f \mu m_{H} c_{\mathrm{s}}}{2 D}\left[\frac{\alpha_{B}}{\pi r L_{\mathrm{LyC}} \sin (\beta)}\right]^{-1 / 2}$,

where $f \approx 1$ is the filling factor for a cylinder of radius $r$ losing mass from one side, $c_{\mathrm{s}} \approx 11 \mathrm{~km} \mathrm{~s}^{-1}$ is the sound speed in photoionized plasma, and $\beta$ is the angle between the jet axis and the direction of the ionizing radiation from a source with luminosity $L_{\mathrm{LyC}}$ located a distance $D$ from the jet. We use the inventory of massive stars and their ionizing photon luminosities cataloged by Smith (2006) to calculate the photoablation rate of the irradiated jet. Given the uncertainty in the three-dimensional structure of the Carina Nebula, we assume that the angle of the incoming radiation is $90^{\circ}$ for all of the jets. Estimated this way, the median mass-loss rate is $\sim 1.4 \times$ $10^{-6} \mathrm{M}_{\odot} \mathrm{yr}^{-1}$. Mass-loss rates estimated from $L_{1}$ for each jet are listed in Table 2.

Comparing mass-loss rates estimated by Smith et al. (2010) from H $\alpha$ with $\dot{M}_{\text {jet }}$ estimated from [Fe II], we find that the latter is $\sim 5$ 100 times higher. The ratio of the $\mathrm{H} \alpha$ - and [Fe II]-derived mass-loss rates for each jet is listed in Table 2 and plotted in Fig. 22.

Fig. 22 shows a comparison of the two methods used to estimate the mass-loss rate based on the detection of near-IR [Fe II]. Mass-loss rates estimated from the requirement that a jet survives to a length $L_{1}$ are higher than those calculated from $n_{\min }$ for all sources. Imposing the more stringent constraint that a jet with density $\geq n_{\min }$ survives to a distance $L_{1}$ requires a higher initial density, and therefore yields a higher mass-loss rate. Even with this accounting, the estimated mass-loss rates are still lower limits. Inferring the jet density from the length of the continuous inner jet assumes that photoablation eventually truncates the jet. However, many jets show evidence for time-variable mass-loss rates (see Section 4.3). In this case, the jet length is a measure of how long the jet has been 'on', provided the density is above $n_{\min }$. A high-density jet that has been losing mass at a high rate for only a short time may have a small length, $L_{1}$, on the sky but support a density much higher than the minimum required to shield $\mathrm{Fe}^{+}$(see Section 4.5).

If we instead assume that mass funnels into the jet in a continuous stream, we can estimate the observed length that a jet would attain before being truncated by photoablation in the $\mathrm{H}$ II region. Taking the lowest mass-loss rate estimate for an $\mathrm{HH}$ jet in Carina, $\dot{M}_{\text {jet }} \sim 10^{-9}$ $\mathrm{M}_{\odot} \mathrm{yr}^{-1}$ for $\mathrm{HH} 1015$ from the $\mathrm{H} \alpha \mathrm{EM}$ (Smith et al. 2010), we expect the jet to extend at least $\sim 310$ arcsec. This exceeds the length of the longest one-sided jet length in the sample ( 308 arcsec, see Table 2). However, the survival of $\mathrm{Fe}^{+}$in all of these jets requires a higher density, and therefore mass-loss rate. Jets with higher massloss rates than assumed in this simple estimate would survive in the $\mathrm{H}_{\text {II }}$ region even longer. Thus, the $[\mathrm{Fe}$ II]-bright jets must reflect a recent change in the mass-loss rate.

\subsection{Implied accretion rate and estimated accretion luminosity}

We list the bolometric luminosity $\left(L_{\mathrm{bol}}\right)$ of the candidate jet driving sources measured by Povich et al. (2011) and Ohlendorf et al. (2012) in Table 3. Povich et al. (2011) classify half of the jet-driving sources as Stage 0/I, reflecting their youth (7/12). HH 666 is the only jet source identified as Class II, although it still has a strong
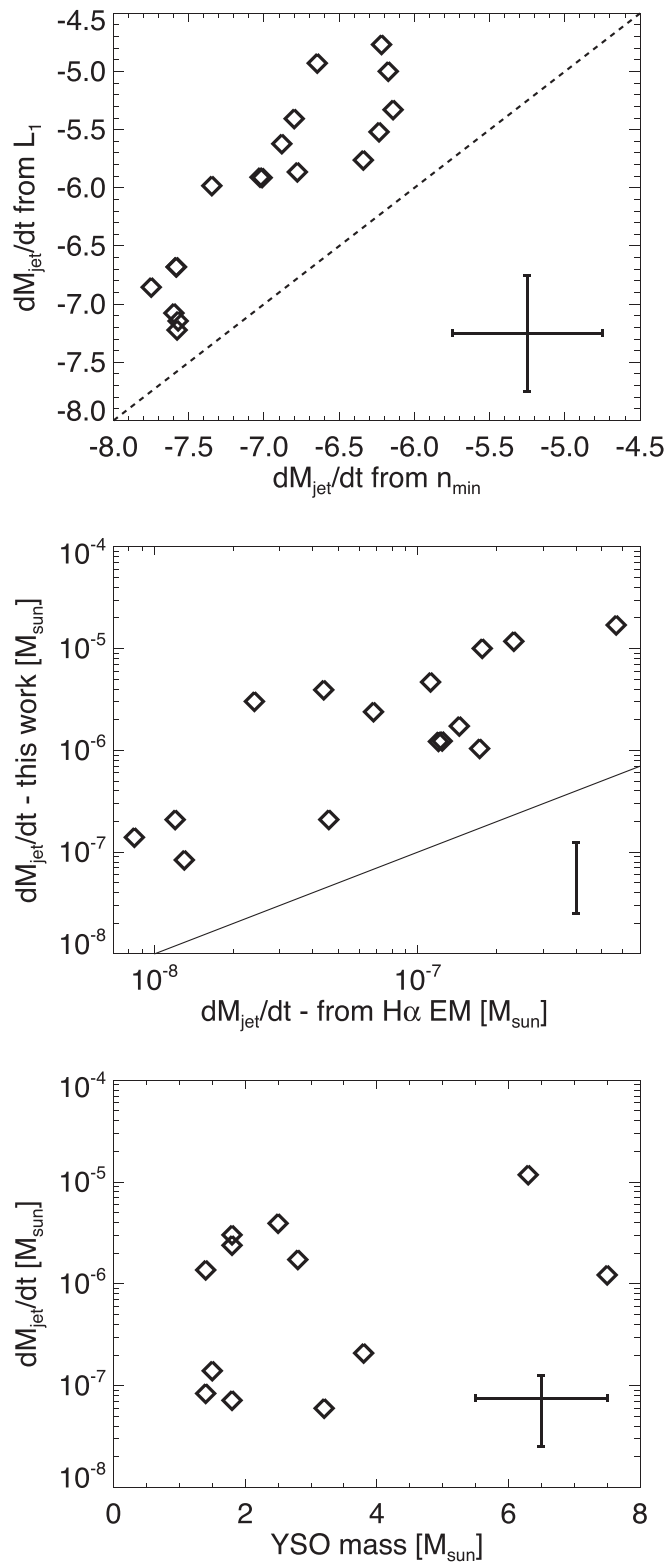

Figure 22. Top: comparison of $\dot{M}_{\text {jet }}$ estimated from the minimum density, $n_{\text {min }}$, to maintain a slow-moving ionization front in the jet and $\dot{M}_{\text {jet }}$ estimated by requiring that the neutral jet core survives to a distance $L_{1}$ in the $\mathrm{H}$ II region (see Section 4.4). The dashed line shows where the two estimates are equal. Middle: comparison of $\dot{M}_{\text {jet }}$ estimated by requiring that the neutral jet core survives to a distance $L_{1}$ in the $\mathrm{H}_{\text {II }}$ region to $\dot{M}_{\text {jet }}$ estimated from the $\mathrm{H} \alpha$ EM by Smith et al. (2010). Bottom: jet mass-loss rate versus the driving source mass Povich et al. (2011, see Table 3). For all three panels, error bars showing the typical uncertainty are shown in the lower right.

IR excess (Smith et al. 2004), indicating that a significant amount of circumstellar material remains. Furthermore, HH 666 is tilted $\gtrsim 30^{\circ}$ away from the plane of the sky (Reiter \& Smith 2014), allowing a clearer view down the envelope cavity opened by the jet. The remaining jet sources have an ambiguous classification of their evolutionary stage (4/12). Two protostars with an ambiguous evolutionary classification can be seen in $\mathrm{H} \alpha$ images (HH 1163 and possibly $\mathrm{HH} 1010$ ), indicating more clearing of the circumstellar environment that suggests more evolved sources (Class II). The remaining jet-driving sources are only seen at IR and longer wavelengths, suggesting earlier evolutionary stages. Four jets that 
Table 3. Jets and their driving sources.

\begin{tabular}{|c|c|c|c|c|c|c|c|c|}
\hline Jet & PCYC & $\log \left(L_{\mathrm{bol}}\right)$ & Mass & Stage & Ohlendorf & $\log \left(L_{b \mathrm{a} o l}\right)$ & Mass & Comment \\
\hline HН 666 & 345 & $3.1(1.7)$ & $6.3(1.3)$ & II & $\mathrm{J} 104351.5-595521$ & $2.6(2.6-3.1)$ & $3.2(1.7-9.2)$ & SBB2004, RS2013. \\
\hline HН 901 & - & - & - & - & - & - & - & \\
\hline НH 902 & - & - & - & - & J104401.8-593030 & - & - & Ruled out by RS2014 proper motions. \\
\hline НH 1066 & 429 & $2.1(1.3)$ & $2.8(1.6)$ & A & J104405.4-592941 & - & - & Identified in RS2013. \\
\hline HH 900 & - & - & - & - & - & - & - & S2013 candidates ruled out by R2015a. \\
\hline HН 903 & - & - & - & - & J104556.4-600608 & $2.4(2.4-2.4)$ & $4.3(4.3-4.3)$ & \\
\hline HH 1004 & 1198 & $3.5(0.9)$ & $7.5(3.0)$ & A & J104644.8-601021 & $2.2(1.9-3.2)$ & $4.6(2.6-7.0)$ & \\
\hline HH 1005 & - & - & - & - & $\mathrm{J} 104644.2-601035$ & $2.4(2.4-2.6)$ & $5.5(5.5-7.3)$ & \\
\hline HH 1006 & 1173 & $1.7(0.3)$ & $1.8(0.9)$ & $0 / \mathrm{I}$ & J104632.9-600354 & - & - & YSO identified by Sahai et al. (2012). \\
\hline HH 1007 & - & - & - & - & - & - & & \\
\hline HH 1010 & 55 & $1.6(1.0)$ & $1.8(1.4)$ & A & J104148.7-594338 & $1.8(1.6-3.1)$ & $1.9(1.3-6.7)$ & \\
\hline HH 1014 & 984 & $2.3(1.4)$ & $2.5(1.8)$ & $0 / \mathrm{I}$ & J104545.9-594106 & - & - & \\
\hline HH 1015 & 538 & $1.3(0.9)$ & $1.5(1.2)$ & $0 / \mathrm{I}$ & - & - & - & \\
\hline $\mathrm{HH}$ c-3 & 760 & $1.3(0.9)$ & $1.4(1.3)$ & $0 / \mathrm{I}$ & J104504.6-600303 & - & - & \\
\hline HН 1159 & - & - & - & - & - & - & - & \\
\hline HН 1160 & 787 & $2.1(1.3)$ & $3.8(1.3)$ & $0 / \mathrm{I}$ & J104509.4-600203 & $2.1(1.7-2.5)$ & $4.3(1.4-6.4)$ & \\
\hline HH 1161 & - & - & - & - & $\mathrm{J} 104509.2-600220$ & - & - & \\
\hline HН 1162 & - & - & - & - & J104513.0-600259 & - & - & \\
\hline HН 1163 & 803 & $1.3(0.2)$ & $1.8(0.9)$ & A & $\mathrm{J} 104512.0-600310$ & - & - & \\
\hline HH 1164 & 790 & $1.9(0.6)$ & $3.2(1.2)$ & $0 / \mathrm{I}$ & - & - & - & \\
\hline HН 1156 & 986 & $1.4(0.9)$ & $1.4(1.1)$ & $0 / \mathrm{I}$ & - & - & - & \\
\hline
\end{tabular}

Notes: SBB2004 = Smith et al. (2004); S2013 = Shinn et al. (2013); RS2013 = Reiter \& Smith (2013); RS2014 = Reiter \& Smith (2014); R2015a = Reiter et al. (2015a).

emerge from dense globules do not have an IR point source detected on the jet axis - HH 901, HH 902, HH 900, and HH 1161.

Many of the putative jet driving sources have luminosities $L_{\mathrm{bol}}$ $\sim 20-200 \mathrm{~L}_{\odot}$, similar to the luminosities observed from low-mass stars in an elevated accretion state (see e.g. table 1 in Hartmann \& Kenyon 1996 and Audard et al. 2014). Reipurth \& Aspin (1997) studied $14 \mathrm{HH}$ jet-driving sources and concluded that most of their sources are consistent with low-mass protostars that are in an elevated accretion phase, similar to FU Orionis outbursts. In outburst, the accretion luminosity will dominate the stellar luminosity, increasing the total observed luminosity by one to two orders of magnitude. Outbursting low-mass stars may therefore be detected in surveys that are not sensitive to quiescent low-mass (and thus low-luminosity) protostars.

To test whether the $\mathrm{HH}$ jet-driving sources in Carina could be low-mass sources in an outburst, we estimate the fraction of $L_{\mathrm{bol}}$ that may be due to accretion. Assuming that $\dot{M}_{\text {jet }}=0.1 \times \dot{M}_{\text {acc }}$, we can estimate the accretion luminosity, $L_{\text {acc }}$. For a $1 \mathrm{M}_{\odot}$ star accreting from $5 \mathrm{R}_{\odot}$, the $L_{\text {acc }}$ implied by the median $\dot{M}_{\text {jet }}$ estimated in Section 4.4 is $\sim 2 \mathrm{~L}_{\odot}$. This $L_{\text {acc }}$ is smaller than $L_{\mathrm{bol}}$ of all the sources presented in this paper (all points in Fig. 23 fall below the one-to-one line). Allowing for a lower outflow efficiency - i.e. if $\dot{M}_{\text {jet }}$ is 1 per cent of $\dot{M}_{\text {acc }}$, rather than 10 per cent - increases the estimated $L_{\text {acc }}$ by an order of magnitude. This brings the estimated $L_{\text {acc }}$ roughly equal to the four lowest luminosity jet-driving sources in our sample (see Table 2).

Conversely, if we assume that accretion dominates the luminosity of a $100 \mathrm{~L} \odot$ protostar, we estimate an accretion rate of $\sim 10^{-5}$ $\mathrm{M}_{\odot} \mathrm{yr}^{-1}$ (again using the parameters for a low-mass protostar, $\left.1 \mathrm{M}_{\odot}, 5 \mathrm{R}_{\odot}\right)$. This is of the order of the $\dot{M}_{\text {acc }}$ implied by the jet if the outflow efficiency $\dot{M}_{\text {jet }} / \dot{M}_{\text {acc }}=0.01$. While this high luminosity and vigorous accretion rate is consistent with observed FU Ori-like sources (Hartmann \& Kenyon 1996; Audard et al. 2014), these outbursts are short lived (typically $\sim 10-100 \mathrm{yr}$ ). Detecting many such sources in a single region is unlikely, even for the large population of low-mass protostars in Carina $(\sim 14000$; see Broos

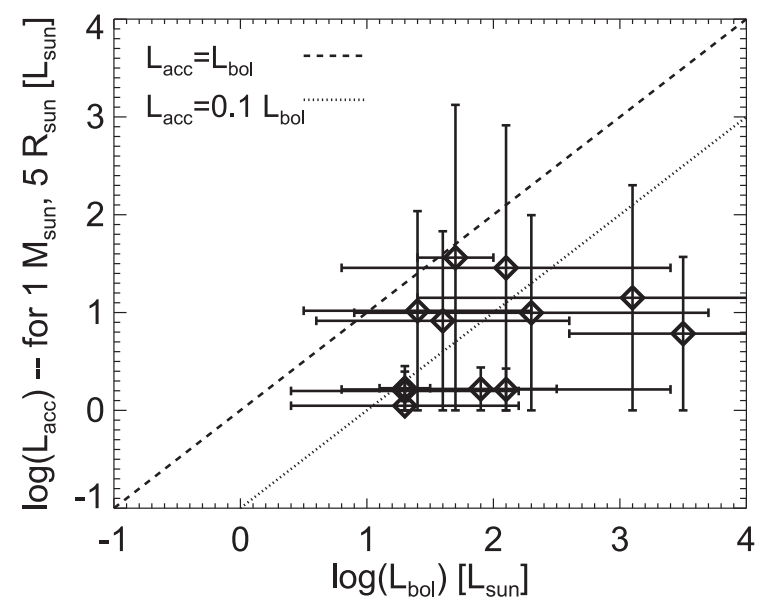

Figure 23. Estimated $L_{\text {acc }}$ derived by assuming $\dot{M}_{\text {acc }}=10 \times \dot{M}_{\text {jet }}$ for a $1 \mathrm{M}_{\odot}$ star accreting from $5 \mathrm{R}_{\odot}$ plotted versus $L_{\text {bol }}$ from the PCYC model fit. The dashed (dotted) line shows where points would fall if $L_{\text {acc }}$ accounted for 100 per cent (10 per cent) of $L_{\text {bol }}$. A higher stellar mass $\left(>1 \mathrm{M}_{\odot}\right)$, more compact accretion geometry $(<5 \mathrm{R} \odot)$, or lower outflow efficiency (1 per cent rather than 10 per cent) will all increase $L_{\text {acc }}$.

et al. 2011), especially given that only $\sim 25$ FU Ori-like sources are known (Audard et al. 2014).

Assuming an FU Ori outburst rate of $8 \times 10^{-4} \mathrm{yr}^{-1} \mathrm{star}^{-1}$ (as Hillenbrand \& Findeisen 2015 infer for Class I sources from the models of Bae et al. 2014), we may detect $\sim 100$ FU Ori-like outbursts among the $\sim 10^{4}$ sources reported by Broos et al. (2011) over a period of $10 \mathrm{yr}$. However, only $\sim 375$ of those sources fall within the area images with WFC3-IR, making it unlikely that we would detect more than $\sim 3$ outbursts. Better estimates that account for the decrease in outburst rate with evolution (i.e. $3 \times 10^{-6} \mathrm{yr}^{-1}$ $\mathrm{star}^{-1}$ for Class II sources) will reduce the likelihood that we have caught a source in outburst. Thus, we consider this possibility to be unlikely. 
For intermediate-mass driving sources, the underlying stellar luminosity is higher, leading to larger bolometric luminosities, even in the quiescent state. $L_{\text {acc }}$ will also increase due to the increased source mass and smaller inner disc radii (see e.g. Muzerolle et al. 2004). Using the estimated masses from Povich et al. (2011) and assuming that all intermediate-mass stars accrete from $3 \mathrm{R}_{\odot}$ (the radius used by Muzerolle et al. 2004), we find a median accretion luminosity of $\sim 24 \mathrm{~L} \odot$. This is similar to the smallest luminosities in our sample (see Table 3), although we note that this estimate requires parameters consistent with intermediate-mass stars to produce this agreement. Altogether, this further supports our argument that the protostars driving the $\mathrm{HH}$ jets in Carina are predominately of intermediate-mass.

\subsection{Momentum injection}

In this paper, we build on the argument first made in Reiter \& Smith (2013) that the HH jets in Carina are the unveiled counterparts to the molecular outflows typically observed from intermediate-mass protostars in embedded regions. Because much of the obscuring gas and dust has been cleared in the $\mathrm{H}$ II region, the core of the atomic jet is illuminated by nearby O-type stars and can be studied directly, rather than inferred from the properties of the outflow it entrains. The existence of two-component outflows from some embedded protostars (e.g. Reiter et al. 2015a,b) supports this interpretation. Another test is to determine whether the $\mathrm{HH}$ jets in Carina have enough momentum to power the molecular outflows observed from intermediate-mass protostars in other regions. Estimates of whether jets from low-mass stars have sufficient momentum to drive molecular outflows provide conflicting results (e.g. Hartigan, Morse \& Raymond 1994; Bacciotti \& Eislöffel 1999).

Reiter \& Smith (2014) showed that the HH jets in Carina have high momentum even though their velocities are similar to jets from low-mass stars because of their high densities. All of the HH jets presented in this paper must also have high densities to shield [Fe II]. Combining the median mass-loss rate, $\sim 1.4 \times 10^{-6} \mathrm{M}_{\odot} \mathrm{yr}^{-1}$, with an assumed velocity, $v_{\text {jet }}=140 \mathrm{~km} \mathrm{~s}^{-1}$, yields a lower limit on the average momentum rate of $\sim 2 \times 10^{-4} \mathrm{M}_{\odot} \mathrm{km} \mathrm{s}^{-1} \mathrm{yr}^{-1}$. Assuming the same velocity for all of the jets in Carina, the range of mass-loss rates estimated for the $\mathrm{HH}$ jets in Carina correspond to momenta rates ranging from $\sim 10^{-5}-10^{-3} \mathrm{M}_{\odot} \mathrm{km} \mathrm{s}^{-1} \mathrm{yr}^{-1}$. This is similar to the range Beltrán et al. (2008) find in their study of the CO outflows from intermediate-mass stars, suggesting that the momenta of these jets is sufficient to power the outflows.

\subsection{Statistics}

In the total area covered by our WFC3-IR images, Povich et al. (2011) detected 55 IR point sources with spectral energy distributions consistent with being intermediate-mass YSOs $\left(2 \mathrm{M}_{\odot} \lesssim M \lesssim\right.$ $\left.10 \mathrm{M}_{\odot}\right)$. These model fits include an estimate of the evolutionary stage of the YSO using a system parallel to the empirical classification of T Tauri stars, although an analogous evolutionary sequence is not yet firmly established for intermediate-mass stars (see discussion in section 3 of Povich et al. 2011). More than half of the detected sources are classified as Stage 0/I/II from the best-fitting SED model - 23/55 or 42 per cent are classified as Stage 0/I, while $12 / 55$ or 22 per cent are Stage II; the remaining 36 per cent have an ambiguous evolutionary stage. Near-IR [Fe II] clearly traces a collimated $\mathrm{HH}$ jet back to 12 of those protostars for a total of 22 per cent of the protostars observed to be associated with jets (note that the HH 903 driving source was not included in the PCYC, see Section 3.2). The jet-driving sources appear to be young with 58 per cent
(7/12) classified as Stage 0/I, 8 per cent (1/12) Stage II sources, and the remaining sources with an ambiguous evolutionary stage (4/12 or 33 per cent). Excluding those sources that can be identified in an $\mathrm{H} \alpha$ image, 75 per cent $(9 / 12)$ of the jet-driving sources remain embedded.

Assuming that every protostar drives an episodic jet, the fraction of intermediate-mass protostars detected within the area imaged with WFC3-IR that are associated with an [Fe II] jet suggests that the jets are 'on' $\sim 25$ per cent of the time. This may be an overestimate given the uneven sensitivity to embedded sources across the survey area. If the sample is contaminated with a few low-mass sources in an elevated luminosity (FU Orionis-like) outburst state, then this number will be even lower. While we cannot exclude the possibility that some of the $\mathrm{HH}$ jets presented here are driven by low-mass sources, it is unlikely to be true for most of them (see Sections 4.4 and 4.5).

\section{CONCLUSIONS}

We present narrow-band, near-IR [Fe II] images obtained with HST/WFC3-IR of 18 jets and two HH objects in the Carina Nebula. Bright [Fe II] emission traces 18 separate collimated bipolar jets. This survey targets jets with a candidate driving source identified near the jet axis. [Fe II] emission connects the larger scale $\mathrm{H} \alpha$ jet to the intermediate-mass protostar that drives it in 13/18 sources. Jets without a detection of their driving source primarily emerge from small, dense globules.

Simultaneous offline continuum images allow us to remove PDR emission from the irradiated surface of the natal cloud and isolate emission from the jet. The dense cores of the jets traced by [Fe II] appear highly collimated, while this is not always the case for $\mathrm{H} \alpha$. In some cases, the two lines appear to trace different outflow components altogether. From these new [Fe II] images, we report the discovery of two new jets, HH 1156 and HH 1164, that cannot be identified as such from $\mathrm{H} \alpha$ images alone.

Bright [Fe II] emission in externally irradiated protostellar jets requires high densities to shield against further ionization in the $\mathrm{H}$ II region. From this minimum density, we estimate high massloss rates that point to powerful jets. With these new and mass-loss rates and conservative estimates of the jet velocity, we find that the momentum of the jets is similar to the outflow momentum measured in molecular outflows from intermediate-mass stars (Beltrán et al. 2008). However, both the assumed velocities and estimated massloss rates are likely to be underestimates. The true jet momentum may be as much as an order of magnitude higher, suggesting that these jets are more than capable of entraining the molecular outflows more typically seen from intermediate-mass protostars.

Altogether, these highly collimated jets look like a scaled-up version of the jets seen from low-mass stars. The harsh UV environment in the Carina Nebula offers a rare glimpse of collimated jets from intermediate-mass protostars. These jets remain invisible in the absence of external irradiation, but may well be a ubiquitous feature of star formation. If so, this offers strong evidence that similar accretion physics governs the formation of stars of all masses.

\section{ACKNOWLEDGEMENTS}

We thank the referee for a thorough and thoughtful report. MR would like to thank Joan Najita and Jaehan Bae for helpful discussions. Support for this work was provided by NASA through grants AR-12155, GO-13390, and GO-13391 from the Space Telescope Science Institute. This work is based on observations made with the NASA/ESA Hubble Space Telescope, obtained from the Data 
Archive at the Space Telescope Science Institute, which is operated by the Association of Universities for Research in Astronomy, Inc., under NASA contract NAS 5-26555. These HST observations are associated with programmes GO 10241, 10475, 13390, and 13391.

\section{REFERENCES}

Alecian E. et al., 2013, MNRAS, 429, 1001

Arce H. G., Goodman A. A., 2001, ApJ, 554, 132

Arce H. G., Shepherd D., Gueth F., Lee C.-F., Bachiller R., Rosen A., Beuther H., 2007, Protostars and Planets V. Univ. Arizona Press, Tucson, AZ, p. 245

Audard M. et al., 2014, Protostars and Planets VI. Univ. Arizona Press, Tucson, AZ, p. 387

Bacciotti F., Eislöffel J., 1999, A\&A, 342, 717

Bachiller R., Guilloteau S., Dutrey A., Planesas P., Martin-Pintado J., 1995, A\&A, 299, 857

Bae J., Hartmann L., Zhu Z., Gammie C., 2013, ApJ, 764, 141

Bae J., Hartmann L., Zhu Z., Nelson R. P., 2014, ApJ, 795, 61

Balbus S. A., Hawley J. F., 1991, ApJ, 376, 214

Bally J., Reipurth B., 2001, ApJ, 546, 299

Bally J., Zinnecker H., 2005, AJ, 129, 2281

Bally J., O’Dell C. R., McCaughrean M. J., 2000, AJ, 119, 2919

Bally J., Licht D., Smith N., Walawender J., 2006, AJ, 131, 473

Bautista M. A., Pradhan A. K., 1998, ApJ, 492, 650

Bautista M. A., Fivet V., Quinet P., Dunn J., Gull T. R., Kallman T. R., Mendoza C., 2013, ApJ, 770, 15

Bautista M. A., Fivet V., Ballance C., Quinet P., Ferland G., Mendoza C., Kallman T. R., 2015, ApJ, 808, 174

Beltrán M. T., Estalella R., Girart J. M., Ho P. T. P., Anglada G., 2008, A\&A, 481, 93

Beuther H., Walsh A. J., Longmore S. N., 2009, ApJS, 184, 366

Bouvier J., Alencar S. H. P., Harries T. J., Johns-Krull C. M., Romanova M. M., 2007, Protostars and Planets V. Univ. Arizona Press, Tucson, AZ, p. 479

Broos P. S. et al., 2011, ApJS, 194, 2

Burrows C. J. et al., 1996, ApJ, 473, 437

Calvet N., Muzerolle J., Briceño C., Hernández J., Hartmann L., Saucedo J. L., Gordon K. D., 2004, AJ, 128, 1294

Carrasco-González C. et al., 2012, ApJ, 752, L29

Deb N. C., Hibbert A., 2010, ApJ, 711, L104

Ellerbroek L. E., Podio L., Kaper L., Sana H., Huppenkothen D., de Koter A., Monaco L., 2013, A\&A, 551, A5

Enoch M. L., Evans N. J., II, Sargent A. I., Glenn J., Rosolowsky E., Myers P., 2008, ApJ, 684, 1240

Evans N. J., II et al., 2003, PASP, 115, 965

Evans N. J., II et al., 2009, ApJS, 181, 321

Ferreira J., Dougados C., Cabrit S., 2006, A\&A, 453, 785

Giannini T. et al., 2015, ApJ, 798, 33

Guzmán A. E., Garay G., Brooks K. J., Voronkov M. A., 2012, ApJ, 753, 51

Haro G., 1952, ApJ, 115, 572

Haro G., 1953, ApJ, 117, 73

Hartigan P., Morse J. A., Raymond J., 1994, ApJ, 436, 125

Hartigan P., Reiter M., Smith N., Bally J., 2015, AJ, 149, 101

Hartmann L., Kenyon S. J., 1996, ARA\&A, 34, 207

Harvey P., Merín B., Huard T. L., Rebull L. M., Chapman N., Evans N. J., II, Myers P. C., 2007, ApJ, 663, 1149

Herbig G. H., 1950, ApJ, 111, 11

Herbig G. H., 1951, ApJ, 113, 697

Hillenbrand L. A., Findeisen K. P., 2015, ApJ, 808, 68

Hinkley S. et al., 2013, ApJ, 763, L9

Hubrig S., Carroll T. A., Schöller M., Ilyin I., 2015, MNRAS, 449, L118

Johns-Krull C. M., Valenti J. A., Hatzes A. P., Kanaan A., 1999a, ApJ, 510, L41

Johns-Krull C. M., Valenti J. A., Koresko C., 1999b, ApJ, 516, 900

Johns-Krull C. M., Valenti J. A., Saar S. H., 2004, ApJ, 617, 1204

Johns-Krull C. M. et al., 2013, ApJ, 765, 11

Johnston K. G. et al., 2015, ApJ, 813, L19
Jørgensen J. K., van Dishoeck E. F., Visser R., Bourke T. L., Wilner D. J., Lommen D., Hogerheijde M. R., Myers P. C., 2009, A\&A, 507, 861

Kraus S. et al., 2010, Nature, 466, 339

Krist J. E. et al., 1998, ApJ, 501, 841

Luhman K. L., Engelbracht C. W., Luhman M. L., 1998, ApJ, 499, 799

McCaughrean M. J. et al., 1998, ApJ, 492, L157

Mendigutía I., Mora A., Montesinos B., Eiroa C., Meeus G., Merín B., Oudmaijer R. D., 2012, A\&A, 543, A59

Muzerolle J., D’Alessio P., Calvet N., Hartmann L., 2004, ApJ, 617, 406

Nussbaumer H., Storey P. J., 1988, A\&A, 193, 327

Ohlendorf H., Preibisch T., Gaczkowski B., Ratzka T., Grellmann R., McLeod A. F., 2012, A\&A, 540, A81

Padgett D. L., Brandner W., Stapelfeldt K. R., Strom S. E., Terebey S., Koerner D., 1999, AJ, 117, 1490

Povich M. S. et al., 2011, ApJS, 194, 14

Preibisch T., Ratzka T., Gehring T., Ohlendorf H., Zinnecker H., King R. R., McCaughrean M. J., Lewis J. R., 2011, A\&A, 530, A40

Quinet P., 1996, A\&AS, 116, 573

Raga A. C., Lora V., Smith N., 2010, Rev. Mex. Astron. Astrofis., 46, 179

Reipurth B., Aspin C., 1997, AJ, 114, 2700

Reipurth B., Bally J., Fesen R. A., Devine D., 1998, Nature, 396, 343

Reiter M., Smith N., 2013, MNRAS, 433, 2226

Reiter M., Smith N., 2014, MNRAS, 445, 3939

Reiter M., Smith N., Kiminki M. M., Bally J., Anderson J., 2015a, MNRAS, 448,3429

Reiter M., Smith N., Kiminki M. M., Bally J., 2015b, MNRAS, 450, 564

Richer J. S., Shepherd D. S., Cabrit S., Bachiller R., Churchwell E., 2000, Protostars and Planets IV. Univ. Arizona Press, Tucson, AZ, p. 867

Rodríguez-Ardila A., Pastoriza M. G., Viegas S., Sigut T. A. A., Pradhan A. K., 2004, A\&A, 425, 457

Sahai R., Güsten R., Morris M. R., 2012, ApJ, 761, L21

Sandell G. et al., 1999, ApJ, 519, 236

Shakura N. I., Sunyaev R. A., 1973, A\&A, 24, 337

Shepherd D. S., Testi L., Stark D. P., 2003, ApJ, 584, 882

Shinn J.-H. et al., 2013, ApJ, 777, 45

Smith R. G., 1987, MNRAS, 227, 943

Smith N., 2002, MNRAS, 331, 7

Smith N., 2006, MNRAS, 367, 763

Smith N., Hartigan P., 2006, ApJ, 638, 1045

Smith N., Bally J., Morse J. A., 2003, ApJ, 587, L105

Smith N., Bally J., Brooks K. J., 2004, AJ, 127, 2793

Smith N., Bally J., Walborn N. R., 2010, MNRAS, 405, 1153

Tan J. C., McKee C. F., 2003, in Burton M., Jayawardhana R., Bourke T., eds, Proc. IAU Symp. 221, Star Formation at High Angular Resolution. Astron. Soc. Pac., San Francisco, p. 274

Vink J. S., Drew J. E., Harries T. J., Oudmaijer R. D., Unruh Y., 2005, MNRAS, 359, 1049

Wade G. A., Bagnulo S., Drouin D., Landstreet J. D., Monin D., 2007, MNRAS, 376, 1145

Ybarra J. E., Barsony M., Haisch K. E., Jr, Jarrett T. H., Sahai R., Weinberger A. J., 2006, ApJ, 647, L159

\section{SUPPORTING INFORMATION}

Additional Supporting Information may be found in the online version of this article:

We present $[\mathrm{Fe} \mathrm{II}]$ ratio tracings, as in Fig. 18, of all jets presented in this work in the online version of this paper:

(http://www.mnras.oxfordjournals.org/lookup/suppl/doi:10.1093/ mnras/stw2296/-/DC1).

Please note: Oxford University Press is not responsible for the content or functionality of any supporting materials supplied by the authors. Any queries (other than missing material) should be directed to the corresponding author for the article.

This paper has been typeset from a $\mathrm{T}_{\mathrm{E}} \mathrm{X} / \mathrm{L} \mathrm{T} \mathrm{E} \mathrm{X}$ file prepared by the author. 